\title{
The Effects of Fungal Pigments on Matrix-Assisted Laser Desorption/Ionization Time-of-Flight Mass Spectral Peak Formation
}

Amanda Dawn Buskirk

West Virginia University

Follow this and additional works at: https://researchrepository.wvu.edu/etd

\section{Recommended Citation}

Buskirk, Amanda Dawn, "The Effects of Fungal Pigments on Matrix-Assisted Laser Desorption/lonization Time-of-Flight Mass Spectral Peak Formation" (2010). Graduate Theses, Dissertations, and Problem Reports. 4566.

https://researchrepository.wvu.edu/etd/4566

This Thesis is protected by copyright and/or related rights. It has been brought to you by the The Research Repository @ WVU with permission from the rights-holder(s). You are free to use this Thesis in any way that is permitted by the copyright and related rights legislation that applies to your use. For other uses you must obtain permission from the rights-holder(s) directly, unless additional rights are indicated by a Creative Commons license in the record and/ or on the work itself. This Thesis has been accepted for inclusion in WVU Graduate Theses, Dissertations, and Problem Reports collection by an authorized administrator of The Research Repository @ WVU. For more information, please contact researchrepository@mail.wvu.edu. 
The Effects of Fungal Pigments on Matrix-Assisted Laser Desorption/Ionization Time-of-Flight Mass Spectral Peak Formation

Amanda Dawn Buskirk

Thesis submitted to the

School of Medicine

at West Virginia University

in partial fulfillment of the requirements

for the degree of

Master of Science

in

Biomedical Sciences

Donald H. Beezhold, Ph.D., Chair

Kathleen M. Brundage, Ph.D.

Slawomir Lukomski, Ph.D.

Rosana Schafer, Ph.D.

Paul D. Siegel, Ph.D.

Department of Microbiology, Immunology, and Cell Biology

Morgantown, West Virginia

2010

Keywords: Mass Spectrometry, MALDI-TOF, Aspergillus, Fungi, Microbial Fingerprinting, Pigment, Melanin 


\begin{abstract}
The Effects of Fungal Pigments on Matrix-Assisted Laser Desorption/Ionization Time-of-Flight Mass Spectral Peak Formation
\end{abstract}

Amanda Dawn Buskirk

\begin{abstract}
Fungi are ubiquitous microorganisms that commonly cause diseases with high mortality rates in immunocompromised hosts. In order to successfully treat the infection and subsequently decrease mortality rates, it is necessary to rapidly identify the causal organism(s) and promptly begin the proper treatment. Common identification methods, such as viable culture characterization, are often time consuming or not species-specific, as is the case with many molecular or immunodiagnostic assays. Matrix-assisted laser desorption/ionization time-of-flight mass spectrometry (MALDI-TOF MS) has been used to discriminate between many clinically relevant microorganisms. Recently, MALDI-TOF MS has been used to discriminate between monilaceous fungal species, however, darkly pigmented fungi yield poor mass spectra with only a few peaks of low relative abundance. Darkly pigmented fungi are of interest because they are common indoor contaminants, and frequently a cause of disease in immunocompromised patients. In this study, the effect of dark fungal pigments on the ionization of standard peptide and protein solutions during MALDI-TOF MS is investigated. Solutions of human serum albumin and angiotensin II were spiked with varying concentrations of both synthetic melanin and fungal pigments extracted from Aspergillus niger, A. fumigatus, and A. terreus. Serum albumin and angiotensin II $[\mathrm{M}+\mathrm{H}]^{+}$signals were suppressed in a concentration dependent manner. Examination of the MALDI sample deposit under magnification showed significant heterogeneity, with regions of highly concentrated pigment appearing black. MALDI-TOF mass spectra acquired from darkly pigmented regions of the sample deposit yielded poor or no $[\mathrm{M}+\mathrm{H}]^{+}$ion signal from the standard. In contrast, nonpigmented regions within the sample deposit and liquid hyphal negative control extracts of A. niger, A. fumigatus, and A. terreus produced no such inhibition. Gas chromatography analysis of each fungal pigment extract revealed the presence of putative cell wall components including aliphatic chains and fatty acids. Field emission scanning electron microscopy (FESEM) showed A. niger and A. fumigatus extracts retain original spore morphology, while the $A$. terreus extract was homogeneous and morphologically similar to synthetic melanin. This study demonstrates that synthetic and fungal pigmentation derived from darkly pigmented fungi inhibits the desorption/ionization process during MALDI-TOF MS, however these fungi may be successfully analyzed by MALDI-TOF MS when culture methods that suppress pigment expression are employed.
\end{abstract}


To my dad, who's constant love, support, and encouragement has carried me further than he will ever know. 


\section{ACKNOWLEDGEMENTS}

Throughout my graduate school experience, I have had the pleasure of working with numerous talented people. I would like to take this opportunity to acknowledge and extend my heartfelt gratitude to all of those who have helped make this thesis possible. First of all, I'd like to thank my advisor, Don Beezhold, for his patience, understanding, and superior guidance throughout my years in his laboratory. He has been an exceptional mentor. I would also like to thank my committee members for their extra time, guidance, and support. Many thanks go out to the members of the ACIB lab at NIOSH. In particular, I owe much gratitude to Justin Hettick, Steve Templeton, and Brett Green, who have never failed to keep me on track with kind words of encouragement and support, and who have taught me valuable lessons that I will carry with me always. Additionally, I would like to thank all the friends I have made throughout my journey in Morgantown. I am blessed to have had the opportunity to meet and form wonderful friendships with many of them. I would also like to take this opportunity to extend my deepest gratitude and appreciation to my parents, Roger and Bonnie. Their continuous support, encouragement, and sacrifices have brought me where I am today and can never be repaid. In addition to my parents, my

family and extended family deserve more acknowledgement and thanks than the words on this paper can illustrate. Their belief in me and my abilities has never ceased to astound me. 


\section{TABLE OF CONTENTS}

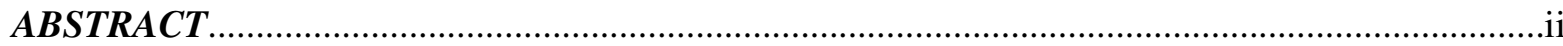

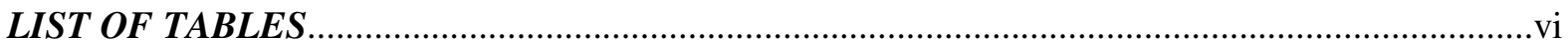

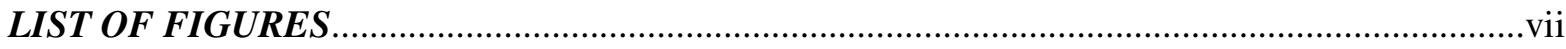

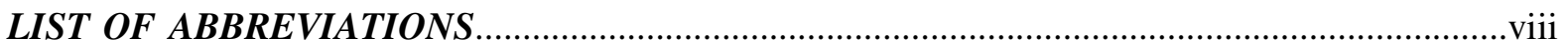

CHAPTER I: GENERAL INTRODUCTION/LITERATURE REVIEW ......................................

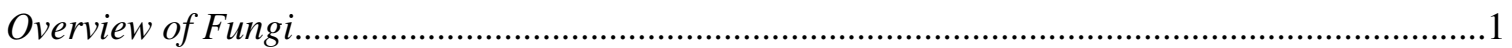

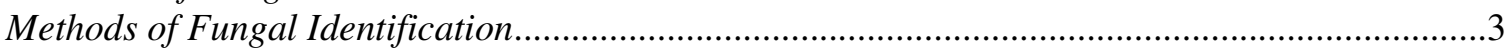

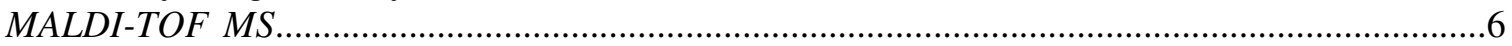

Microbial Discrimination/Identification using MALDI-TOF MS ..............................................

Limitations of MALDI-TOF MS Microbial Fingerprinting .........................................................11

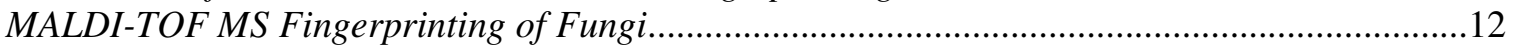

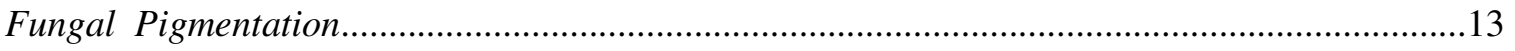

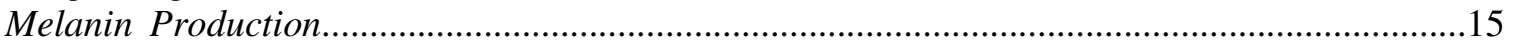

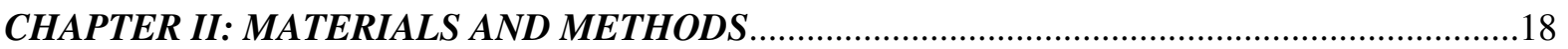

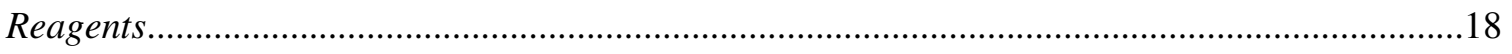

Fungal Culture ............................................................................................................................

Preparation of Fungal Culture Samples for MALDI-TOF MS Analysis......................................19

Preparation of Synthetic Melanin Samples for MALDI-TOF MS Analysis...................................19

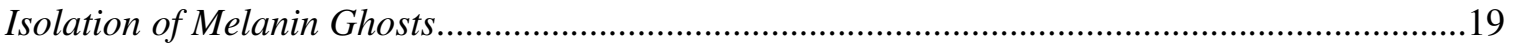

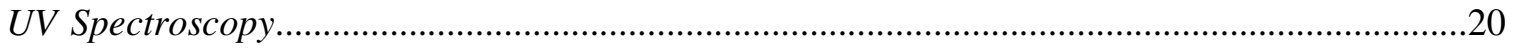

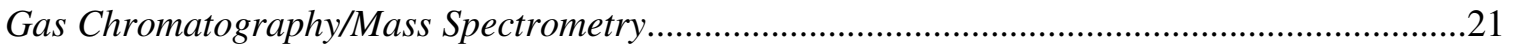

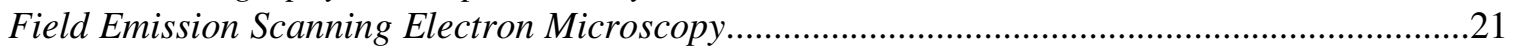

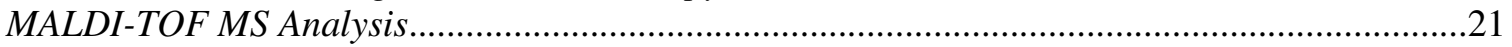

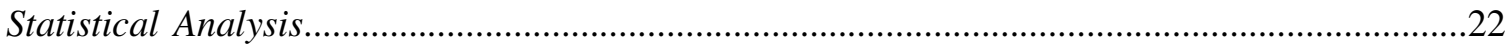

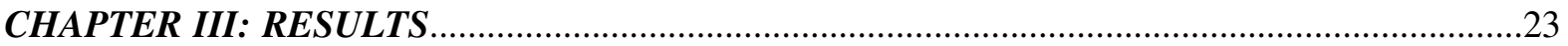

MALDI-TOF MS Suppression by Darkly Pigmented Fungi.......................................................23

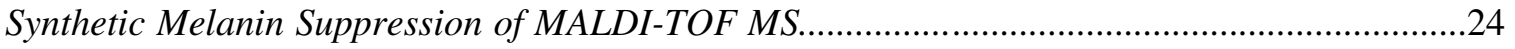

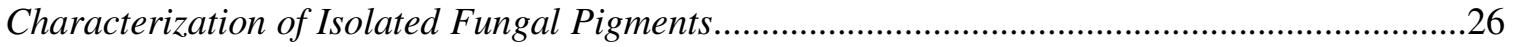

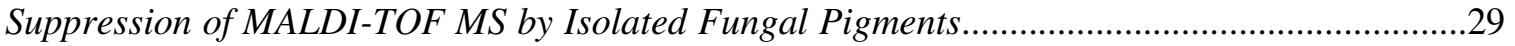

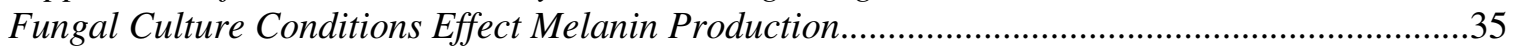

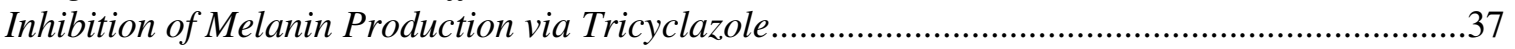

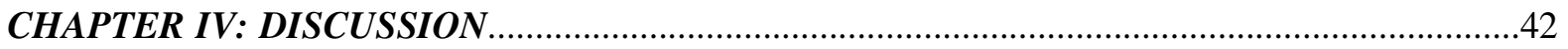

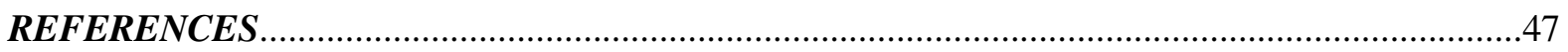

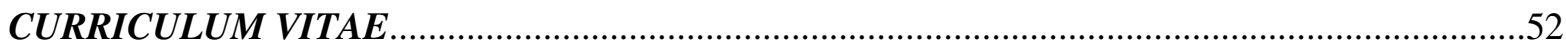




\section{LIST OF TABLES}

Table

Page

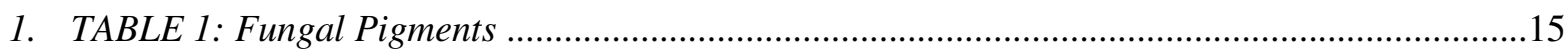




\section{LIST OF FIGURES}

Figure

Page

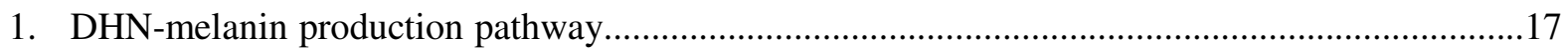

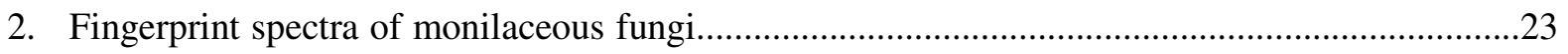

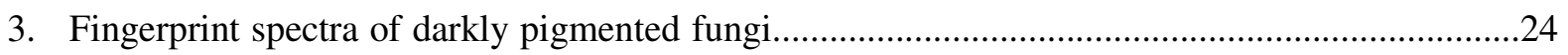

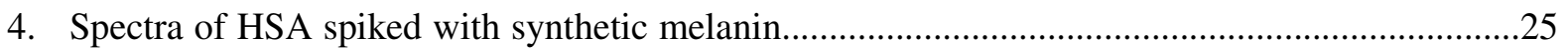

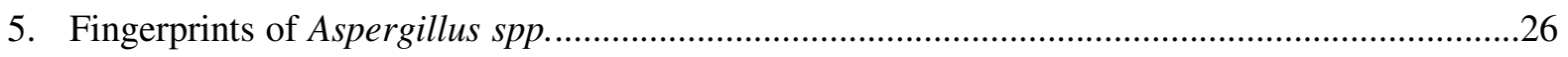

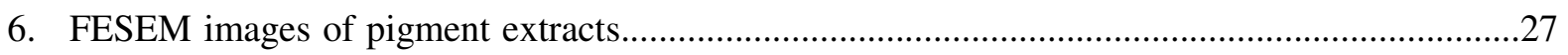

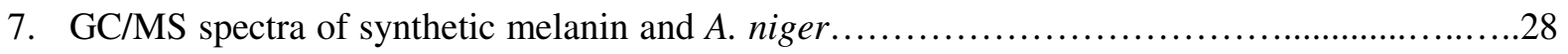

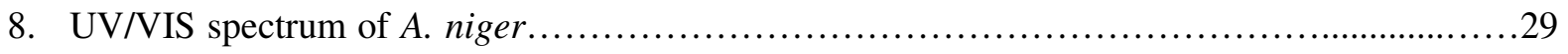

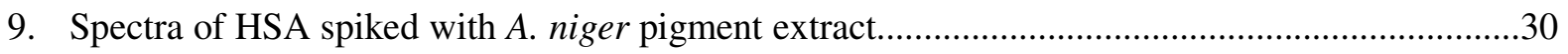

10. Spectra of HSA spiked with A. fumigatus pigment extract.........................................................31

11. Spectra of HSA spiked with A. terreus pigment extract.......................................................... 32

12. Graph of effect of fungal pigments on HSA peak height............................................................33

13. Light microscopy images of MALDI target spot deposition.........................................................34

14. MALDI Q-TOF mass spectra of angiotensin II spiked with A. fumigatus pigment extract............35

15. Fingerprints of Aspergillus spp grown in submerged cultures....................................................36

16. 2D canonical distribution of fungi grown in submerged cultures.................................................37

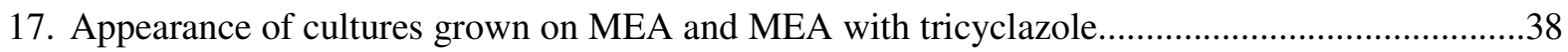

18. Fingerprints of $A$. niger 7 days vs. 14 days, MEA vs. MEA with tricyclazole...............................39

19. Fingerprints of $A$. fumigatus 7 days vs. 14 days, MEA vs. MEA with tricyclazole........................40

20. Fingerprints of A. terreus 7 days vs. 14 days, MEA vs. MEA with tricyclazole.............................41 


\section{LIST OF ABBREVIATIONS}

\begin{tabular}{|c|c|}
\hline $\mathrm{ACN}$ & Acetonitrile \\
\hline $\mathrm{CHCA}$ & $\alpha$-cyano-4-hydroxycinnamic acid \\
\hline $\mathrm{CSF}$ & Cerebral spinal fluid \\
\hline CT & Computer tomography \\
\hline DDI & Deionized water \\
\hline DHN & Dihydroxynapthalene \\
\hline DOPA & Dihydroxyphenylalanine \\
\hline ELISA & Enzyme-linked immunosorbent assay \\
\hline $\mathrm{EtOH}$ & Ethanol \\
\hline FESEM & Field emission scanning electron microscopy \\
\hline GC/MS & Gas chromatography/Mass spectrometry \\
\hline HSA & Human serum albumin \\
\hline IA & Invasive aspergillosis \\
\hline ITS & Internal transcribed spacer \\
\hline MALDI & Matrix-assisted laser/desorption ionization \\
\hline MEA & Malt extract agar \\
\hline MEB & Malt extract broth \\
\hline \multirow[t]{2}{*}{ MIQE } & Minimum Information for Publication of Quantitative Real-Time PCR \\
\hline & Experiments \\
\hline MRI & Magnetic resonance imaging \\
\hline MRSA & Methicillin-resistant Staphylococcus aureus \\
\hline MS & Mass spectrometry \\
\hline MWCO & Molecular weight cutoff \\
\hline PBS & Phosphate buffered saline \\
\hline
\end{tabular}




$\begin{array}{ll}\text { PCR } & \text { Polymerase chain reaction } \\ \text { Q-TOF MS } & \text { Quadrupole time-of-flight mass spectrometry } \\ \text { TCA } & \text { The Citric Acid Cycle } \\ \text { TFA } & \text { Trifluoroacetic acid } \\ \text { TOF } & \text { Time-of-flight } \\ \text { UV } & \text { Ultraviolet } \\ \text { VIS } & \text { Visible }\end{array}$




\section{Chapter I}

General Introduction/Literature Review

\section{Overview of Fungi}

Fungi are a diverse kingdom of spore-forming microorganisms. Fungal spores emerge from the reproductive structures (e.g. hyphae and conidial head) of germinating fungi (1). These spores are released into the environment upon environmental disturbances including wind, vibrations, humidity, and water exposures (2). Because of their small size and aerodynamic nature, these spores may be carried for long distances in the wind current until they deposit onto a suitable substrate under appropriate environmental conditions. These substrates include nutrients such as carbohydrates, sugars, and high water content, with environmental temperatures commonly within the range of $25-37^{\circ} \mathrm{C}$. This means that fungi can grow on many surfaces especially in indoor environments that are prone to water infiltration, contain cellulose-based building materials, and lack adequate ventilation $(1,2)$.

Because of the ability to easily propagate large cultures of fungi, as well as the ease in manipulating the culture conditions to produce desirable products, many fungal products have been utilized in industry, agriculture, and pharmaceuticals. Saccharomyces cerevisiae, an ascomycetes yeast, has been used in the fermentation process to produce alcohol, yogurt, and bread (3). Several fungi, such as members of the genera Aspergillus, Chaetomium, and most commonly known Penicillium, are used to produce antimicrobial drugs from metabolites and toxins produced during fungal growth (4). Furthermore, mycotoxins from the fungi Aspergillus terreus and Penicillium citrinum have been used to produce lovastatin and compactin, respectively, which are drugs used to lower cholesterol in patients (5).

Despite the many beneficial uses of fungi, personal exposure to spores, hyphae, and fungal fragments can result in adverse health effects such as induction and exacerbation of allergy and asthma, as well as causing mycoses or toxicoses in immunocompromised patients (6-9). Eduard et al. reported that the total number of spores within contaminated buildings may exceed levels of $10^{7} \mathrm{spores} / \mathrm{m}^{3}$, and those spores are commonly inhaled by humans during natural and occupational fungal exposures $(2,10)$. Health 
effects occurring in patients following fungal exposures range from fatigue, respiratory symptoms, gastrointestinal problems, as well as neurocognitive symptoms, dizziness, and vision problems (11). It has been estimated that as much as $10 \%$ of the U.S. population has allergy to common fungal agents (11). In addition, different climatic regions of the world support varying assemblages of fungal species and the concentration of these fungi are dependent upon the season (12). In 2002, it was estimated that in the U.S. alone, 10,000 lawsuits related to mold in households were pending in the court systems. Insurance companies had paid out over 2 billion dollars in Texas alone due to mold related claims, illustrating the economic impact of unwanted fungal growth (11).

To date, research efforts have focused on understanding the health effects associated with fungal exposures, such as allergic sensitization, toxicoses, invasive infections, and exacerbation of existing pulmonary conditions such as allergy and asthma $(1,6-9,13,14)$. Immunocompetent individuals rapidly clear inhaled fungal spores and fragments with no damage to the lungs (15). However, in immunosuppressed, neutropenic individuals, fungi can begin to germinate within 6-8 hours after exposure $(1,16)$. Once the fungus begins to grow within the airways of these patients, invasive infection most commonly ensues and is associated with high mortality rates ranging between 50-99\%, even after strong antifungal treatments $(1,11,16)$. Due to increasing numbers of immunosuppressed patients, fungal infections have placed a significant economic burden on health care. Invasive aspergillosis (IA), most commonly caused by Aspergillus fumigatus, is one of the predominant diseases associated with immunocompromised patients. It was reported to occur in $17-26 \%$ of patients after lung transplants, 5$15 \%$ after allogenic bone marrow transplants, $5-24 \%$ of those with acute leukemia, and $2-13 \%$ of patients after heart transplants in the U.S. (11). The average age of patients with invasive aspergillosis is 53 years old, and 58\% of patients are men. In 1996 alone, there were over 10,000 hospitalizations for invasive aspergillosis, with greater than $19 \%$ mortality in those individuals. These cases resulted in over 170,000 days of hospitalization, costing the U.S. over 630 million dollars, further illustrating the economic burden of fungal exposures (11). 


\section{Methods of Fungal Identification}

Successful treatment of fungal infections is dependent upon rapid identification of the causal fungus followed by the administration of appropriate antifungal agents $(17,18)$. Samples must first be obtained from the patient to identify the causal organism(s) and may be obtained from the tissue, blood/serum, bronchoalveolar lavage fluid (BAL), or cerebrospinal fluid (CSF). Obtaining tissue samples or biopsies from a patient that is immunosuppressed is an invasive procedure that may be detrimental to the patient, thus this method is often avoided. Therefore, fluids are most commonly used to determine the contaminating organism(s). Viable culture is the most widely used method for identifying fungal infections from the patient (19). This method involves culturing the causal fungus on differential media selective for specific fungi and then macro and microscopic examination of the culture by a trained mycologist. Morphology, hyphal fragmentation, colony color, and spore emergence from the hyphae are colony characteristics that can be used to identify fungal colonies. Major drawbacks with these identification methods are that the laboratory requires a trained mycologist and a long culture incubation time (24-72 hours for most fungi, with some requiring weeks for growth). Further, many fungi may have similar morphological characteristics that may lead to misidentification (20).

While the 'gold standard' for positive fungal identification in clinical samples commonly requires positive culture identification, as well as visualization of fungal masses via chest $\mathrm{x}$-rays, computer tomography (CT scans), or magnetic resonance imaging (MRI), other techniques have also been used to in conjunction with these methods $(1,19)$. Most of these are immunoassays or enzyme-linked immunosorbent assays (ELISAs), which use monoclonal and polyclonal antibodies that have been developed for detecting fungal components or metabolites that may be present in the serum of the patient. In these cases serum is tested by direct or indirect immunoassay formats for the presence of previously identified and characterized antigens isolated from a number of known fungal pathogens (21-26). Some common monoclonal antibodies include those against $\beta$-glucans, ergesterol, glycoproteins, mannans, or other cell wall components. In addition, putative diagnostic markers have been identified for species- 
specific antigenic proteins in common fungal pathogens, such as Asp $\mathrm{f} 1$ from Aspergillus fumigatus, the causal organism of IA $(27,28)$.

Since IA is a disease primarily associated with immunosuppressed patients, and is associated with high mortality rates, it is of major concern in the medical community. IA is difficult to diagnose in the early stages, however, at later stages antifungal therapy often fails resulting in mortality rates as high as $99 \%(1,11,29,30)$. There are two commercially available immunoassays for Aspergillus identification, both with varying specificity and sensitivity. These assays, the Platelia Aspergillus enzyme immunoassay and the Pastorex Aspergillus latex agglutination test, detect galactomannan, a carbohydrate cell wall component, associated with Aspergillus, and utilize the same rat monoclonal antibody, EBA-2 (26). In the Pastorex assay, latex beads are coated with the EBA-2 antibody and the patient serum is added to the beads. If the sample is positive for galactomannan, then the beads will agglutinate. The Platelia assay is a sandwich ELISA, in which a 96-well polystyrene plate is first coated with the EBA-2 antibody, the patient serum is added, and an enzyme-linked EBA-2 detection antibody is added to the well. Specific substrate for the enzyme-linked detection antibody is then added to the well and a color change is observed in a positive sample (22). Both of these techniques can yield a false positive if the patient has been treated with $\beta$-lactam antibiotics. These antibiotics are isolated from Penicillium, and because the EBA-2 antibody specifically recognizes the $(1 \rightarrow 5)-\beta{ }_{-\mathrm{D}}$-galactofuranoside side chains of the galactomannan moiety, which are found in Aspergillus, Penicillium, and Paecilomycese fungi, false positives may occur. A false positive may also arise if the origin of the patient infection is from Penicillium or Paecilomycese for the same reason $(21,30,31)$. Therefore, these assays are limited to only identifying known antigens. Patient samples containing antigens from an unknown pathogen may also result in false negatives. Though ELISAs can be completed rapidly in comparison to viable culture and morphological characterization, many of the antibodies developed to date are not species-specific. Therefore, despite the possibility of rapidly identifying the presence of fungus in the patient sample, more species-specificity is needed. 
Polymerase Chain Reaction (PCR) is a molecular technique that has recently been used for detecting pathogenic and non-pathogenic fungi. This method involves using commercially available primers directed toward common sequences within the fungal genome, typically from the 18S rRNA subunit, though others, such as primers directed towards the genes encoding ergosterol, $\beta$-glucans, $\beta$ tubulin, or other cell wall components may also be used $(19,32)$. The latter primers can identify a positive fungal infection but are not species-specific, as many cell wall components are conserved in most fungi, regardless of the species origin (32). Internal transcribed spacer regions (ITS regions) are also targets for PCR amplification, as they are often conserved among species $(19,32)$. Primers directed toward the ITS regions are species-specific in most cases, offering as high as $98 \%$ identification, with low levels of false positives (19). Real-time PCR for fungal identification has also been used. This technique offers the ability to quantitate the number of fungal specific gene copies, which has been proposed to be correlated to disease progression. This information would be invaluable for disease prognosis, as well as for identifying the proper course of prophylactics to use for the best patient outcome (19).

Though PCR offers increased sensitivity for fungal identification, approaching $100 \%$ sensitivity and specificity when done in conjunction with other methods, there are many drawbacks to this technique $(23,24)$. The major issues surrounding PCR for fungal identification are the ubiquitous nature of fungi. Fungi may contaminate samples at the isolation stage, DNA extraction stage, the sample PCR preparation stage, as well as cross-contamination between PCR runs, and therefore are common areas yielding false positives. The presence of fungal components in the DNA extraction solutions, many of which may contain enzymes that have been isolated from different fungi, may also be a source for false positives. Also, many of these solutions are not compatible with common methods for fungal decontamination, such as UV irradiation, phenol precipitation, or filtration (32). Although using PCR using primers directed toward the rRNA subunits can detect the target from a fraction of one organism, interference with the larger amounts of human DNA in the patient samples has been reported. Some of the primers may interact with human DNA, preventing the fungal target from being amplified in high enough amounts to be analyzed. Furthermore, molecular techniques have not been standardized and have only recently been 
realized by the Minimum Information for Publication of Quantitative Real-Time PCR Experiments (MIQE) guidelines $(26,32)$.

Commercially available nucleic acid hybridization probes have also been employed for fungal identification $(19,33)$. In this method, species-specific chemiluminescent single stranded DNA probes are bound to a chip, the organism is cultured and a small amount removed and lysed to release the nucleic acids, which are then denatured and added to the chip. The complementary ribosomal RNA released from the isolated fungus will then hybridize to the probes and activate the chemiluminescence. The degree of hybridization can be measured in relative light units using a luminometer. This technique has high sensitivity and specificity for certain fungi tested, such as Blastomyces dermatitidis, Histoplasma capsulatum, and Coccidiodes immitis, however, cross reactivity with the B. dermatitidis probe has been reported (19). Despite the potential of this technique, the lack of species-specific DNA probes highlights a major drawback in the field of fungal research. Over 70,000 fungal species have been identified, and over 1.5 million species are thought to exist; however, only a fraction of these fungi have been sequenced (34, 35). Further research needs to be completed with this technique to further identify species-specific probes, as well as to increase the commercial availability to realize the potential of this method.

\section{MALDI-TOF MS}

Matrix-assisted laser desorption/ionization time-of-flight mass spectrometry (MALDI-TOF MS) is a more recent analytical method that can be used to identify an analyte present in a sample, and has been used to discriminate between clinically relevant microorganisms $(36,37)$. MALDI is a specific type of ionization method for mass spectrometry that utilizes samples mixed with a matrix, or an $\mathrm{H}^{+}$donor which co-crystallizes with the sample on a target chip. Commonly, the target chip is made of an electrical conductor, such as stainless steel or gold (38). Once the sample is loaded into the mass spectrometer, a UV laser (commonly 337-nm $\mathrm{N}_{2}$ or 355-nm Nd:YAG) is fired at the sample spot. Upon laser excitation of the sample, matrix and co-crystallized analyte are desorbed in a supersonic plume of ions and neutrals 
into the gas phase in the vacuum of the mass spectrometer where transfer of protons from the sample matrix to the analyte occurs $(39,40)$.

Time-of-flight mass spectrometry (TOF MS) is the method used for detecting and analyzing samples $(41,42)$. There is a short source region near the sample plume, which consists of a backing plate and an extraction grid. Upon laser excitation of the sample, the potential energy (PE) of the short source region is zero. A positive voltage is then applied to the backing plate, creating an electric field, thereby increasing the PE "behind" the sample. Ions are accelerated to a constant kinetic energy by the high electrical potential (typically as high as $20 \mathrm{kV}$ ) into a vacuum tube, or field-free drift region. A vacuum tube is used to lessen the potential for collisions within the field free region, as such interactions may alter the velocity of the ions, thereby altering mass resolution of the ions. Mass resolution is the ability to distinguish between peaks within a spectrum based on analyte peak widths as well as relative separation of peaks. The analytes travel through the vacuum tube at a rate that is proportional to their mass to charge ratio, i.e. larger, heavier molecules will travel slower than smaller, lighter molecules, while an increase in the number of charges on an ion will allow it to travel faster than an ion with fewer charges. The ions then impact a detector at the end of the vacuum tube, creating a cascade of electrons which are amplified and detected as electrical current $(41,42)$. The spectra are recorded based on mass/charge $(\mathrm{m} / \mathrm{z})$ and the relative abundance of the analyte (proportional to the number of hits reaching the detector at a specific $m / z)$. Therefore, most protein-containing samples can be analyzed if the protein content is above that of the detectable limit of the instrument. Each protein mixture yields a spectrum that is representative of the molecules within the sample and is unique to individual analytes.

\section{Microbial Discrimination/Identification using MALDI-TOF MS}

MALDI-TOF MS has been widely used for the discrimination and identification of numerous microbes because the spectra produced by MALDI-TOF MS analysis are unique to each microbe, synonymous to a human fingerprint. Thus, these spectra are referred to as microbial "fingerprints". The majority of experiments done using MALDI-TOF MS for the discrimination of microbes have focused on 
bacterial fingerprinting. In 1994, the first experiment using MALDI-TOF MS for bacterial discrimination was able to detect water soluble surface proteins of bacteria, which allowed for the differentiation between gram positive and gram negative organisms (43). Further studies were done to show the ability of MALDI-TOF MS to distinguish between multiple organisms of different species using whole cell bacterial preparations to obtain unique fingerprints for each bacterium (44-46). Briefly, a small aliquot of bacteria were placed on a target spot, allowed to air dry, and then overlaid with matrix before analyzing with the mass spectrometer. Using this technique, many clinically relevant bacteria have been analyzed, including Escherichia coli, Pseudomonas (45), Staphylococcus, Mycobacterium (44), and Bacillus anthracis (46). The ability to differentiate these organisms is due to the presence of unique ions at different $\mathrm{m} / \mathrm{z}$ ranges for each species. A complete list of bacteria which have been analyzed using MALDI-TOF MS is shown in a recent publication by Giebel et al. and includes 22 clinically relevant bacterial genera (47).

MALDI-TOF MS has also been used to differentiate between different strains of the same organism $(48,49)$. For example, this technique has been useful in discriminating between pathogenic, multi-drug resistant strains of normal microbiota, such as Staphylococcus and methicillin resistant Staphylococcus aureus (MRSA) (48). In this study, each strain yielded a unique distinct spectrum, however, when analyzing large numbers of clinical isolates and laboratory strains, the authors state that a distinctive spectrum representative of MRSA could not be obtained. The authors noted, however, that the MRSA strains could be differentiated from each other, and this could be used to identify the origin of clonal strains.

Several studies have focused on the use of MALDI-TOF MS for bacterial identification in clinical laboratories (50-54). In these studies, bacteria had been recovered from blood, cerebrospinal fluid (CSF), pus, biopsy, the respiratory tract, stool, and wounds. Samples were identified using standard phenotypical methodology by using differential growth mediums and conditions. In parallel, a single colony of the bacterium were deposited on a MALDI-TOF MS target plate and overlaid with matrix and then analyzed. (53). Of the 1660 isolates, from 45 genera and 109 species obtained, MALDI-TOF MS was able to 
correctly identify $95.5 \%$ of the organisms, the same as done by standard routine identification, with $84.1 \%$ of the samples being identified at the species level. Forty-six of the isolates were unable to be fingerprinted using MALDI-TOF MS, however, this number was not statistically different from the standard routine identification methods, which failed to identify thirty-two of the isolates. Furthermore, this study reported that the lack of identification by MALDI-TOF MS was related to the lack of reference spectra for comparison of those species. For example, for the bacterial species that contained more than 5 reference spectra, greater than $85 \%$ of those bacterial species were identified using MALDI-TOF MS. This highlights the importance of having a large database library for direct comparison of unknown samples. The authors also reported a cost/time of identification comparison analysis, whereby they compared the standard protocols for bacterial identification with MALDI-TOF MS discriminate analysis. They reported that mass spectrometry identification took approximately 8.5 minutes and cost 1.43 euros/sample. When compared to gram staining, which takes 6 minutes and costs 0.6 euros/sample, API system identification which takes 1080-2880 minutes and costs 4.6-6.0 euros/sample, antibiotic susceptibility testing which takes 1080-2880 minutes and costs 6.6-7.4 euros/sample, Phoenix system identification and susceptibility testing which takes 300-1200 minutes and costs 12.65 euros/sample, and the Vitek system which takes 300-480 minutes for identification and costs 5.9-8.23 euros/sample and in conjunction with susceptibility testing costs 10.38-12.71 euros/sample, MALDI-TOF MS is the most cost and time efficient method for comparable bacterial identification (53).

Further studies have examined the potential use of MALDI-TOF MS for bacterial identification in positive blood cultures. In a study done by Christner et al., 304 positive blood cultures (reported positive by an automated incubation system), of which 277 contained a single bacterium, were analyzed using MALDI-TOF MS fingerprinting (51). The authors report 95\% correct bacterial identification, with $94 \%$ of those samples being identifiable on the same day as the blood samples showed positive culture. Another study of 1,371 isolates obtained from clinical samples were identified using conventional methods and analyzed via MALDI-TOF MS in parallel, reported $95.1 \%$ correct identification rate. In this study 63 isolates were unable to be identified, however, the authors reported possible reasons for the 
misidentification/inability to identify as being initial conventional misidentification in 7 isolates, and the remaining 56 isolates were predominantly misidentified as a result of human error in taxonomic assignment of the reference spectra in the database, recent changes in taxonomy of a given species, or different levels of precision in identification between conventional identification methods and MALDITOF MS (50). A study done in 2010 reported $95.1 \%$ correct species identification of 327 isolates taken from blood, pus, respiratory tract, stool, wounds, CSF, and urine (54).

Another study, which focused on bacterial identification in positive blood cultures, reported the ability of MALDI-TOF MS to identify false positives (as labeled positive by standard conventional protocols), and reported $83.3 \%$ species level identification of gram-negative bacteria, $96.6 \%$ genus level in a sample population of 318 positive blood cultures $(47,52)$. In gram-positive bacteria, species identification was $31.8 \%$ and $65.7 \%$ at the genus level when compared to conventional methods. The comparable difference between identification in gram-positive and gram-negative bacteria may be due to the sample processing done by the authors, as there were multiple wash steps before analysis. Further, gram-positive bacteria have a thicker cell wall, which strongly binds surface proteins and may be harder to lyse. Therefore, the amount of protein obtained from gram-positive bacteria may have been lower than that obtained from the gram-negative bacteria which may have lysed during the washing steps. These are speculations, as the authors did not report sample extraction optimization.

As shown by Hettick et al., bacterial samples with thicker cell walls, such as Mycobacterium spp., are sufficiently discriminated from each other when harsher protein extraction steps are used for sample preparation (55). In this study, several different species of Mycobacterium, including M. fortuitum, M. kansasii, M. intracellulare, M. avium, M. bovis BCG, and M. tuberculosis H37Ra, were subjected organic/acid extraction and analyzed via MALDI-TOF MS. Using linear discriminant analysis of 77 peaks identified among all species of the sample, $100 \%$ of the spectra were correctly identified.

In addition to fingerprinting and discriminating bacteria, MALDI-TOF MS has been used to identify the presence of viruses. Because the standard methodology for viral identification is relatively quick and has a low false identification rate, MALDI-TOF MS has not been as extensively examined as 
an alternative method of viral identification, but has been used for detection. Specifically, 6 different viruses have been fingerprinted including, Heptatitis B and C, Norovirus, Avian influenza viruses, Newcastle disease virus, and the Sindbis virus (47). Commonly, specific proteins or peptides are targeted for specific viral identification. These include the capsid protein (56), fusion proteins $(57,58)$, and glycoproteins associated with the viral envelope (59). In addition, DNA has also been used to specifically identify some viruses, including determining the molecular weight of specific restriction fragments from complementary DNA (60-62).

\section{Limitations of MALDI-TOF MS Microbial Fingerprinting}

Though multiple studies have demonstrated the ability of MALDI-TOF MS to discriminate between numerous clinically relevant microbes, there are also several limitations to this technique. The major limitation is the reference library or database (53). In order to correctly identify clinical pathogens by MALDI-TOF MS, a representative comparison spectrum must be present in the database. If no reference spectrum is present to compare the sample spectrum to, then no identification can be made. This limitation is not one of the instrument, but rather of the compiled database. Therefore, a large, extensive database of all clinically relevant organisms must be compiled for MALDI-TOF MS to correctly identify or discriminate the clinical organism.

In addition, the culture conditions may alter the spectrum of the sample organism when compared to the database. The length of time the culture is grown, as well as the temperature, and the culture medium have been shown to alter the protein content of many microbes (63). The method for protein extraction, where applicable, may also alter the proteins present in the sample. Though intact cell mass spectrometry may not be altered by protein extraction protocols, results from our laboratory show that bead beating samples, both bacterial (Mycobacterium) and fungi (Aspergillus and Penicillium), increase the number of peaks in a spectrum, presumably due to the release of intracellular proteins due to bead beating $(49,64,65)$. In addition, the choice of solvent used for the elution of extracellular proteins must be kept uniform, as different proteins may or may not solubilize in the presence of different organic 
and/or acidic solvents. Therefore, samples comprising the reference database must be treated in the same manner as the unknown sample in order to accurately identify the unknown microbe.

Furthermore, studies have shown that a minimum of $1 \times 10^{4}$ microbial cells must be present within the sample to yield a spectrum with an adequate number of peaks with a high enough relative abundance above the background noise (66). The number of required microbial cells may differ between each individual mass spectrometer used, as each commercially available instrument has differing sensitivities, as well as between microorganisms, as the concentration of soluble proteins may differ between species.

Another limitation of MALDI-TOF MS is the reproducibility of the technique. Reproducibility within a laboratory is often very high (75\% and higher) $(48,67-70)$, however, when the same samples are sent to other laboratories for the same analysis, the reproducibility may decline (as low as 23\%) (71). The lack of reproducibility between laboratories is attributed to the differences in sensitivity of the different mass spectrometers used in each different laboratory. To address this issue, many mass spectrometer companies have been designing 'biotypers', which are essentially mass spectrometers that use a commercial database consisting of a library of spectra from common microorganisms for reference and discrimination (72). These instruments can be used to identify pathogenic organisms from human samples within hours.

\section{MALDI-TOF MS Fingerprinting of Fungi}

MALDI-TOF MS has been used to fingerprint and discriminate between fungi. Fourteen medically important fungal genera have been fingerprinted to date. The first study to fingerprint fungi was published in 2000, where various species of Penicillium, as well as Scytalidum dimidiatum and Trichophyton rubrum were characterized. In most studies examining the fingerprints of fungi, methods to lyse the spores of fungi are used to obtain a fingerprint with a greater number of peaks/ increased relative abundance $(64,65,73)$. These methods include acidic lysing, as done with Saccharomyces cerevisiae (74), or bead beating in an organic/acidic solvent, as done with several species of Aspergillus and 
Penicillium $(64,65)$. In the studies done by Hettick et al., MALDI-TOF MS was able to discriminate between 12 species of Penicillium and 12 species of Aspergillus with a $0 \%$ error rate using discriminant analysis. MALDI-TOF MS was also able to correctly discriminate between 5 different strains of A. flavus $95 \%$ of the time.

Several studies have demonstrated the utility of MALDI-TOF MS for fingerprint profiling of multiple species of fungi; however, one limitation has been identified by several groups $(64,73,75)$. Darkly pigmented fungi, such as Aspergillus niger, Stachybotrys chartarum, and Alternaria alternata could not be fingerprinted using this technique. These samples produce a mass spectrum containing a paucity of distinct peaks preventing identification. Many factors may explain the difficulty in analyzing these darkly pigmented fungi. Although the precise mechanism(s) by which the MALDI desorption/ionization event occurs is the subject of much discussion and active research (39), it is possible that the unique properties of fungal pigments could interfere in a number of fundamental ways with the MALDI process. One plausible explanation for the observed inhibition of MALDI-TOF ionization is that melanin in fungal samples (particularly at high concentrations) directly competes with the organic acid matrix for irradiating photons. Following the MALDI laser pulse, ionization in the dense plume above the sample deposit will only occur where there is a sufficient population of electronically excited matrix molecules. If a photoexcitation/pooling mechanism for MALDI is assumed (76), mobile excitons in the bulk sample deposit could be quenched by fungal pigments.

\section{Fungal Pigmentation}

Darkly pigmented fungi are dark in color because of the high levels of intracellular pigment. Fungi are common plant pathogens, and therefore produce pigmentation to protect themselves from environmental stresses such as ultraviolet light, as well as to aid in the buildup of turgor pressure which the fungi use to penetrate plant tissues (77-79). In submerged conditions, however, most fungi do not produce pigments, presumably because the nutritional needs are altered in these environments and the 
stresses necessary to induce pigment formation are not induced (29). The most common pigment associated with fungi is melanin $(78,79)$.

Melanin is a large, complex macromolecule that is formed through the oxidation of phenolic or indolic compounds. Typically, melanins appear black, brown, or green in color $(79,80)$. Several types of melanins have been identified (Table 1) $(78,79)$. Each type of melanin is produced through the polymerization of different biomolecules, and thus potentially different biosynthetic pathways (79). Phaeomelanins are derived from tyrosine and cysteine, allomelanins are formed from nitrogen-free precursors, and eumelanins are a product of the polymerization of tyrosine, quinones, and free radicals (Table 1) (80). The predominant types of melanin found in fungi are phaeomelanins and eumelanins. In many pathogenic fungi, eumelanins are the most common form of melanin present, though multiple types of melanin may exist simultaneously (78-82). There are also two different associated forms of melanin, including cell-associated or vesicle-associated melanins (78). Commonly, in darkly pigmented fungi, the melanins produced are intercalated within the spore wall of the organism and help to provide stability and strength to the spore wall coating. These melanins are covalently bound to carbohydrates and lipids that make up the cell wall $(16,78)$.

The exact structure of melanin has not been identified due to the properties of this macromolecule which make it difficult to use common biochemical analyses. These properties include: insolubility in organic or aqueous solvents, resistance to degradation by strong acids and alkaline agents, and resistance to bleaching by strong oxidizing agents. Melanins are negatively charged and hydrophobic $(78,80,82$, 83). More importantly, fungal melanins have photoprotective properties whereby they absorb UV light and dissipate it as heat (84-86). This specific property may be at least partially responsible for the inability to acquire unique fingerprints in darkly pigmented fungi. Therefore, it is being hypothesized that the presence of dark pigmentation in darkly pigmented fungi prevents proper ionization of the proteins/peptides (analytes) within the sample preparation, thereby inhibiting peak formation within the spectrum. 


\begin{tabular}{|c|c|c|c|c|}
\hline \multicolumn{5}{|c|}{ Fungal Melanins } \\
\hline Types of fungal melanins & Chemical metabolism & Color & $\begin{array}{c}\text { Family } \\
\text { Occurrence }\end{array}$ & Ref. \\
\hline Eumelanin & $\begin{array}{l}\text { formed through the } \\
\text { polymerization of } \\
\text { tyrosine, quinones, and } \\
\text { free radicals }\end{array}$ & $\begin{array}{l}\text { Black, } \\
\text { dark } \\
\text { green, } \\
\text { dark } \\
\text { brown }\end{array}$ & Various fungi & $(78,80,84)$ \\
\hline $\begin{array}{c}\text { L-3,4- } \\
\text { Dihydroxyphenylalanine } \\
\text { (DOPA) }\end{array}$ & a subtype of eumelanin & ----- & -------- & (78) \\
\hline Phaeomelanin & $\begin{array}{l}\text { formed through the } \\
\text { polymerization of } \\
\text { tyrosine, cysteine, and } \\
\text { dihydroxyphenylalanine } \\
\text { (DOPA) }\end{array}$ & $\begin{array}{l}\text { Red, } \\
\text { yellow }\end{array}$ & Various fungi & $(78,80)$ \\
\hline Allomelanin & $\begin{array}{l}\text { formed from nitrogen-free } \\
\text { precursors }\end{array}$ & $\begin{array}{l}\text { Black, } \\
\text { brown }\end{array}$ & Various fungi & $(80,87)$ \\
\hline Catechol & $\begin{array}{l}\text { a subtype of allomelanin } \\
\text { formed through phenol } \\
\text { oxidation of catechol }\end{array}$ & $\begin{array}{l}\text { Black, } \\
\text { dark } \\
\text { brown }\end{array}$ & Basidiomycetes & $(78,87)$ \\
\hline $\begin{array}{c}1,8- \\
\text { Dihydroxynapthalene } \\
\text { (DHN) }\end{array}$ & $\begin{array}{l}\text { formed from acetyl } \\
\text { coenzyme A and malonyl } \\
\text { coenzyme } \mathrm{A} \text { in the } \\
\text { pentaketide biosynthesis } \\
\text { pathway }\end{array}$ & $\begin{array}{l}\text { Black, } \\
\text { dark } \\
\text { green, } \\
\text { dark } \\
\text { brown }\end{array}$ & $\begin{array}{c}\text { Ascomycetes, } \\
\text { Ascomycetous } \\
\text { imperfecti } \\
\text { fungi, and } \\
\text { Dithideaceous } \\
\text { species }\end{array}$ & (78) \\
\hline $\begin{array}{l}\gamma \text {-glutaminyl-3,4- } \\
\text { dihydroxybenzene }\end{array}$ & $\begin{array}{l}\text { formed through oxidation } \\
\text { of } \gamma \text {-glutaminyl-4- } \\
\text { hydroxybenzene in } \\
\text { shikimic acid pathway }\end{array}$ & $\begin{array}{l}\text { Black, } \\
\text { dark } \\
\text { brown }\end{array}$ & Basidiomycetes & $(78,88)$ \\
\hline
\end{tabular}

Table 1: Common types of fungal melanins.

\section{Melanin Production}

Eumelanins are divided into subgroups including L-3,4-dihydroxyphenylalanine (L-DOPA) melanin and 1,8-dihydroxynapthalene (DHN) melanins (77). Both types of melanin have been identified in fungi, and may exist within the same organism. Though multiple types of melanins are associated with 
fungi, it has been reported that the predominant forms of fungal-associated melanins are DHN-melanins (89). The DHN-melanin synthesis pathway begins with glucose, a common sugar which is used by cells in the citric acid cycle (TCA). Before entering the TCA cycle one molecule of glucose is converted into fructose 6-phosphate, followed by conversion into one molecule of fructose-1,6-diphosphate. This is then converted into glyceraldehyde-3-phosphate and then pyruvate (Figure 1). Pyruvate is converted into two molecules of acetyl coenzyme A (acetyl-CoA) which is the initial molecule for the pentaketide synthesis pathway. In this pathway, acetyl-CoA is converted into 1,3,6,8-4-hydroxynapthalene $(1,3,6,8-4 \mathrm{HN})$. A tetrahydroxynapthalene reductase then converts 1,3,6,8-4-hydroxynapthalene into scytalone. Tricyclazole, an antifungal agent, can block the activity of this reductase producing a shunted pigmented byproduct called flaviolin. Following conversion to scytalone, a trihydroxynapthalene reductase converts scytalone into vermelone. Again, this conversion may be specifically blocked by tricyclazole, producing another shunted pigmented byproduct called 2-hydroxyjugalone. Vermelone is then converted by an unknown convertase into dihydroxynapthalene (DHN) which then is polymerized to produce DHN-melanin (90). Thus, it is being hypothesized that due to the electro- and biochemical nature of melanins present in darkly pigmented fungi, specifically DHN-melanins and/or DHN-melanin-like pigments, are interfering with the proper ionization of the analytes present in the MALDI-TOF MS sample, thereby inhibiting the formation of unique, discriminate fingerprint spectra from these species. 


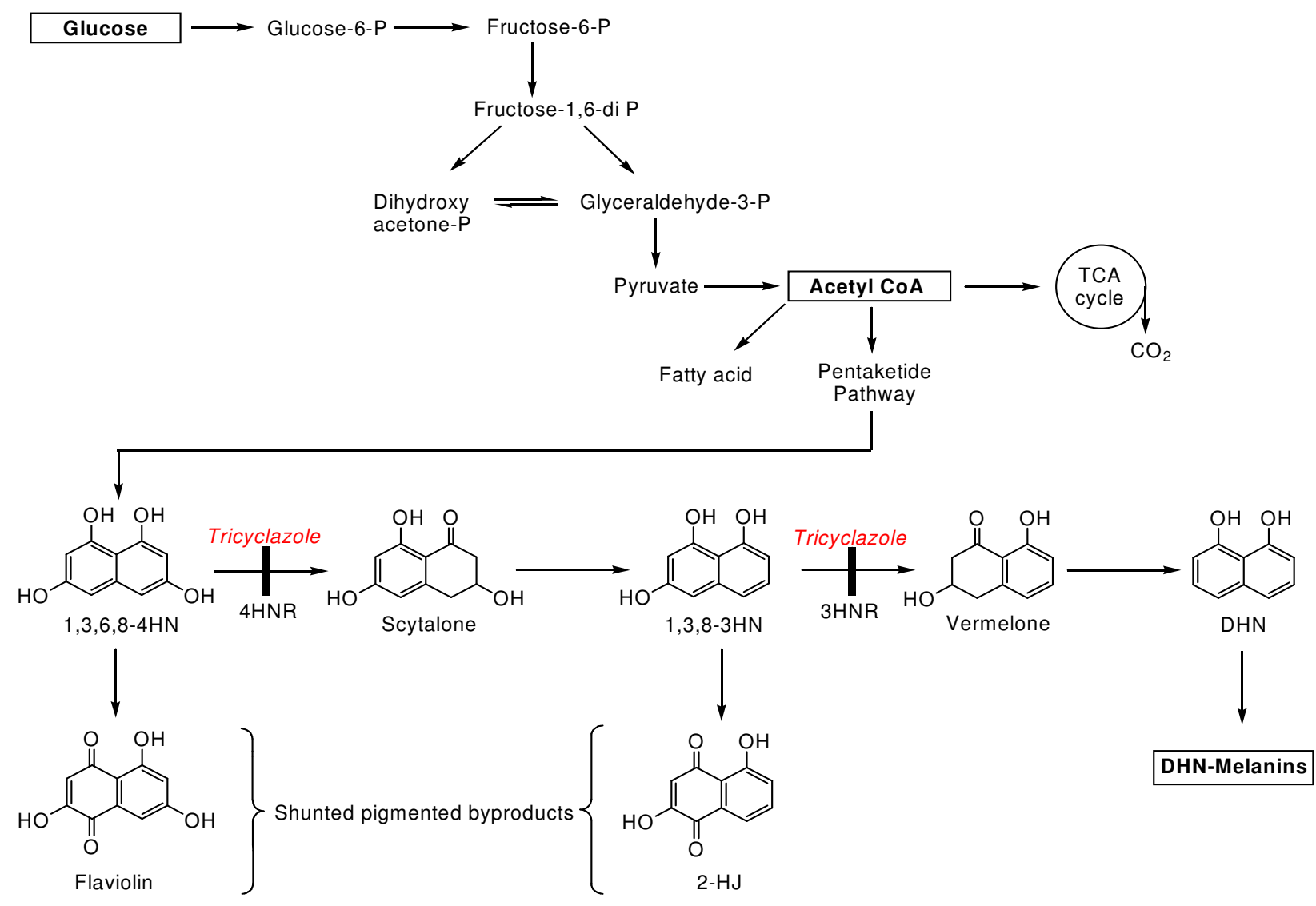

Figure 1: Dihydroxynapthalene melanin (DHN-melanin) production pathway. Figure adapted from Lee et al. (90).

In this study, it is shown that synthetic melanin, as well as fungal pigment extracts from Aspergillus niger, A. fumigatus, and A. terreus, inhibit MALDI-TOF MS analysis of the $66,000 \mathrm{~m} / \mathrm{z}$ peak formation of human serum albumin in a concentration dependent manner. Additionally, heavily pigmented areas within a MALDI-TOF target spot directly inhibit mass spectral formation when compared to an area within the same spot that contains less pigment. Finally, when darkly pigmented fungi, such as A. niger, are cultured under conditions that inhibit pigment formation, a unique fingerprint spectrum is obtained using MALDI-TOF MS. 


\section{Chapter II}

Materials and Methods

\section{Reagents}

Acetonitrile (ACN) (HPLC grade), sodium citrate, and hydrochloric acid (6N) were acquired from Fisher Scientific (Fairlawn, NJ). Tricyclazole was acquired from Wako Pure Chemical Industries, Ltd. (Osaka, Japan). Malt extract agar (MEA) was acquired from Difco (Sparks, MD). Deionized water (DDI) was produced with a Synthesis A-10 (Millipore, Billerica, MA). All other reagents were acquired from Sigma Aldrich (St. Louis, MO) and all reagents were used without further purification.

\section{Fungal Culture}

Fungal isolates were obtained from either the American Type Culture Collection (ATCC, Manassas, VA) or the Fungal Genetics Stock Center (FGSC, Kansas City, MO). Aspergillus terreus (ATCC 1012), A. fumigatus (AF293; FGSC A1100) and A. niger (FGSC A1143) were subcultured from stock sources and grown for 7 or 14 days at $25^{\circ} \mathrm{C}$ on malt extract agar (MEA) nutrient media in $100 \mathrm{~mm}$ culture plates. To ensure reproducibility, each fungal species was cultured eight times independently.

For submerged culture preparation, approximately $1 \times 10^{2}$ spores were inoculated into $50 \mathrm{~mL}$ of malt extract broth (MEB) and grown for 4 days in a $\mathrm{C} 24 \mathrm{KC}$ refrigerated incubator shaker (Edison, NJ) at $25^{\circ} \mathrm{C}$. Cultures were transferred to $50 \mathrm{~mL}$ conical tubes and centrifuged at $41 \mathrm{x}$ g for 5 minutes. The supernatant was retained and frozen at $-80^{\circ} \mathrm{C}$. The mycelial mass was washed 3 times with phosphate buffered saline (PBS) to remove residual MEB and forced through a $70 \mu \mathrm{m}$ mesh filter (BD, Bedford, MA) using a syringe plunger in $20 \mathrm{~mL}$ of PBS. This solution was centrifuged at $41 \mathrm{x}$ g for 5 minutes and the supernatant collected, lyophilized and the dry weight recorded for quantification. The resulting dry mass was suspended in $1 \mathrm{~mL}$ of ACN/4\% TFA (50:50). To ensure reproducibility, each fungal species was cultured eight times independently. For tricyclazole mediated melanin inhibition, cultures were grown for 
7 or 14 days at $25^{\circ} \mathrm{C}$ on MEA plates supplemented with $50 \mu \mathrm{g} / \mathrm{ml}$ of tricyclazole in $1 \%$ ethanol. To ensure reproducibility, each fungal species was cultured eight times independently.

\section{Preparation of Fungal Culture Samples for MALDI-TOF MS Analysis}

Conidia and hyphae derived from one culture plate of A. terreus, A. fumigatus, and A. niger $\left(\sim 10^{8}\right.$ spores/mL) were transferred to $100 \mu \mathrm{L}$ of $0.1 \mathrm{~mm}$ zirconium beads (Biospec, Bartlesville, OK, USA) and $1 \mathrm{~mL}$ of ACN/4\% trifluroacetic acid (TFA) (50:50). The samples were then subjected to three-one minute bead beating cycles. Samples were cooled on ice between bead beating cycles. The resulting solution was centrifuged at $21,600 \times \mathrm{g}$ for ten minutes, and the supernatant fluid retained. A $10 \mu \mathrm{L}$ aliquot of supernatant fluid was combined with $10 \mu \mathrm{L}$ of $\alpha$-cyano-4-hydroxycinnamic acid (CHCA, $10 \mathrm{mg} / \mathrm{mL})$ in 50/50 ACN/0.1\% TFA and duplicate $1.5 \mu \mathrm{L}$ aliquots were deposited on a gold sample stage (Bio-Rad, Hercules, CA) and allowed to air dry prior to MALDI-TOF MS analysis.

\section{Preparation of Synthetic Melanin Samples for MALDI-TOF MS Analysis}

Samples were prepared by dissolving varying concentrations of synthetic melanin over the range of $0.001 \mathrm{mg} / \mathrm{mL}$ to $20 \mathrm{mg} / \mathrm{mL}$ in $50 / 50 \mathrm{ACN} / \mathrm{DDI}$. A $10 \mu \mathrm{L}$ aliquot of synthetic melanin solution was combined with an equal volume of either human serum albumin $(7.5 \mathrm{pmol} / \mu \mathrm{L})$ or angiotensin II $(10$ $\mathrm{pmol} / \mu \mathrm{L})$. A $10 \mu \mathrm{L}$ aliquot of the resultant mixture was combined with $10 \mu \mathrm{L}$ of $\mathrm{CHCA}(10 \mathrm{mg} / \mathrm{mL}$ in 50/50 ACN/0.1\% TFA and duplicate $1.5 \mu \mathrm{L}$ aliquots were deposited on a gold sample stage (Bio-Rad, Hercules, CA) and allowed to air dry prior to MALDI-TOF MS analysis.

\section{Isolation of Melanin Ghosts}

So called "melanin ghosts" were extracted from A. terreus, A. fumigatus, and A. niger using a method modified from Wang et al. (91). Briefly, cultures were grown on MEA for 10 days at $25^{\circ} \mathrm{C}$. Sporulating fungal cultures were harvested from the culture plate using sterilized DDI and the resulting suspension was centrifuged at $1000 \mathrm{x} \mathrm{g}$ for 10 minutes. The pellet was resuspended in $0.1 \mathrm{M}$ sodium 
citrate buffer containing $10 \mathrm{mg} / \mathrm{mL}$ of cell lysing enzymes from $T$. harzianum and $2 \mathrm{mg} / \mathrm{mL}$ cellulase from $T$. reesei and incubated with agitation overnight with at $30^{\circ} \mathrm{C}$. The sample was then pelleted by centrifugation (1000 x g for 10 minutes), washed with PBS and resuspended in $4 \mathrm{M}$ guanidine isothiocyanate and incubated at room temperature (RT) overnight with agitation. The sample was then

pelleted, washed with PBS and resuspended in $1 \mathrm{mg} / \mathrm{mL}$ of proteinase $\mathrm{K}$ in DDI and incubated overnight at $37^{\circ} \mathrm{C}$ with agitation. The sample was pelleted, washed with $\mathrm{PBS}$ and resuspended in $6 \mathrm{M} \mathrm{HCl}$ and boiled for one hour. The sample was then pelleted, washed with PBS and dialyzed against DDI for 10 days at $4^{\circ} \mathrm{C}$ using 3500 molecular weight cutoff (MWCO) dialysis membrane tubing (Spectra/Por ${ }^{\circledR}$, Laguna Hills, CA). The sample was lyophilized and the resulting melanin ghosts used for further experiments.

Melanin ghosts from each Aspergillus species were diluted with ACN/4\% TFA and mixed with an equal volume of $7.5 \mathrm{pmol} / \mu \mathrm{L}$ HSA. A $10 \mu \mathrm{L}$ aliquot of the resulting solution was mixed with $10 \mu \mathrm{L}$ of $10 \mathrm{mg} / \mathrm{mL}$ CHCA and $1.5 \mu \mathrm{L}$ deposited on a gold sample stage (Bio-Rad, Hercules, CA) and allowed to air dry. Each sample was spotted in duplicate.

\section{UV Spectroscopy}

Spectrophotometric characterization of melanin was carried out using a Beckman DU 800 Spectrophotometer (Fullerton, CA) equipped with a deuterium (UV) and a tungsten (Vis) lamp. Data acquisition and processing was carried out using the DU800 Spectrometer software. The analytical wavelength range was 200 to $800 \mathrm{~nm}$. Temperature control was achieved by using a VWR Scientific (Niles, IL) circulating water bath. Fungal pigments were dissolved in $50 / 50 \mathrm{HNO}_{3} / \mathrm{HF}$ solution with sonication for $5 \mathrm{~min}$. Blanking of the instrument was performed with $50 / 50 \mathrm{HNO}_{3} / \mathrm{HF}$ before absorbance readings of the melanin were taken. 


\section{Gas Chromatography/Mass Spectrometry}

Samples were analyzed using an Agilent 6890 gas chromatograph coupled to an Agilent 5975C mass spectrometer (MS) using a 30 m HP5-MS column (Agilent Technologies, Santa Clara, CA). Samples were injected $(1 \mu \mathrm{L})$ at a $20: 1$ split injection ratio into a $300^{\circ} \mathrm{C}$ inlet. Analytes were eluted from the column using $1.0 \mathrm{~mL} / \mathrm{min}$ helium and an oven temperature profile as follows: $50^{\circ} \mathrm{C}$ for $5.0 \mathrm{~min}$ then ramped at $5.0^{\circ} \mathrm{C} / \mathrm{min}$ to a final temperature of $300^{\circ} \mathrm{C}$. The MS source temperature was maintained at $230^{\circ} \mathrm{C}$, the quadrupole temperature was maintained at $150^{\circ} \mathrm{C}$.

\section{Field Emission Scanning Electron Microscopy}

A small aliquot of lyophilized extracted pigment was deposited on double-stick carbon tape on an aluminum mount and sputter coated with gold/palladium. Images were collected on a Hitachi (Tokyo, Japan) S-4800 field emission scanning electron microscope.

\section{MALDI-TOF MS Analysis}

Protein MALDI-TOF mass spectra were acquired using a Ciphergen PBS-IIc linear TOF mass spectrometer (Bio-Rad, Hercules, CA) with a flight path of $0.8 \mathrm{~m}$, capable of mass resolution $(\mathrm{m} / \Delta \mathrm{m})$ of 1000 and mass accuracy of $\pm 1000 \mathrm{ppm}$. Spectra were acquired over the $\mathrm{m} / \mathrm{z}$ range of 0 to $100 \mathrm{~m} / \mathrm{z}$, with delayed extraction parameters set to optimally focus the 10 - to $20-\mathrm{m} / \mathrm{z}$ range. Composite mass spectra are the average of 90 laser shots taken from 20 distinct positions across the sample deposit. These positions were held constant for all samples used in this study, and 337-nm $\mathrm{N}_{2}$ laser pulse energy was maintained just above the threshold for ion production. Mass spectra were externally calibrated using a set of peptide and protein calibrants that covered a range of 1 to $66 \mathrm{~m} / \mathrm{z}$. Peptide MALDI-TOF mass spectra were acquired using a MALDI-SYNAPT ${ }^{\mathrm{TM}}$ MS (Waters, Milford, MA) quadrupole time-of-flight mass spectrometer (Q-TOF MS) capable of mass resolution $(\mathrm{m} / \Delta \mathrm{m})$ of 14,000 and a mass accuracy of $\pm 5 \mathrm{ppm}$. Spectra were acquired over the $\mathrm{m} / \mathrm{z}$ range of 50 to 2,000 Da. Composite mass spectra are the result of a 60 second acquisition with the $\mathrm{N}_{2}$ laser $(337-\mathrm{nm}$ ) operating at $20 \mathrm{~Hz}$, with the laser pulse energy 
maintained just above the threshold for ion production. Mass spectra were externally calibrated using tandem mass spectrometry (MS/MS) data covering the mass range 70 to $1570 \mathrm{M} / \mathrm{z}$.

\section{Statistical Analysis}

Differences in mean peak heights and the effects of fungal strain and pigment level on MALDITOF MS data were statistically determined. Univariate analyses were performed to ensure all data met analytic assumptions. Mixed linear models were used to determine if there were differences in the mean peak height between concentrations within each strain, between pigment levels across all strains, and between strains. Results were considered significantly different at an alpha level of 0.05 . All data were analyzed using SAS 9.1 (SAS Institute, Cary, NC). 


\section{Chapter III}

Results

\section{MALDI Suppression by Darkly Pigmented Fungi}

In the initial studies demonstrating the utilization of MALDI-TOF MS in fungal fingerprinting, closely related fungi of several different species belonging to the genera Aspergillus and Penicillium were differentiated $(64,65)$. It was observed that MALDI-TOF MS fingerprinting produced unique spectra for each species, and sufficiently allowed for reproducible discrimination between five different isolates of $A$. flavus (64). Unpublished results from additional experiments using different fungi also yielded unique spectra. (Figure 2) (92).
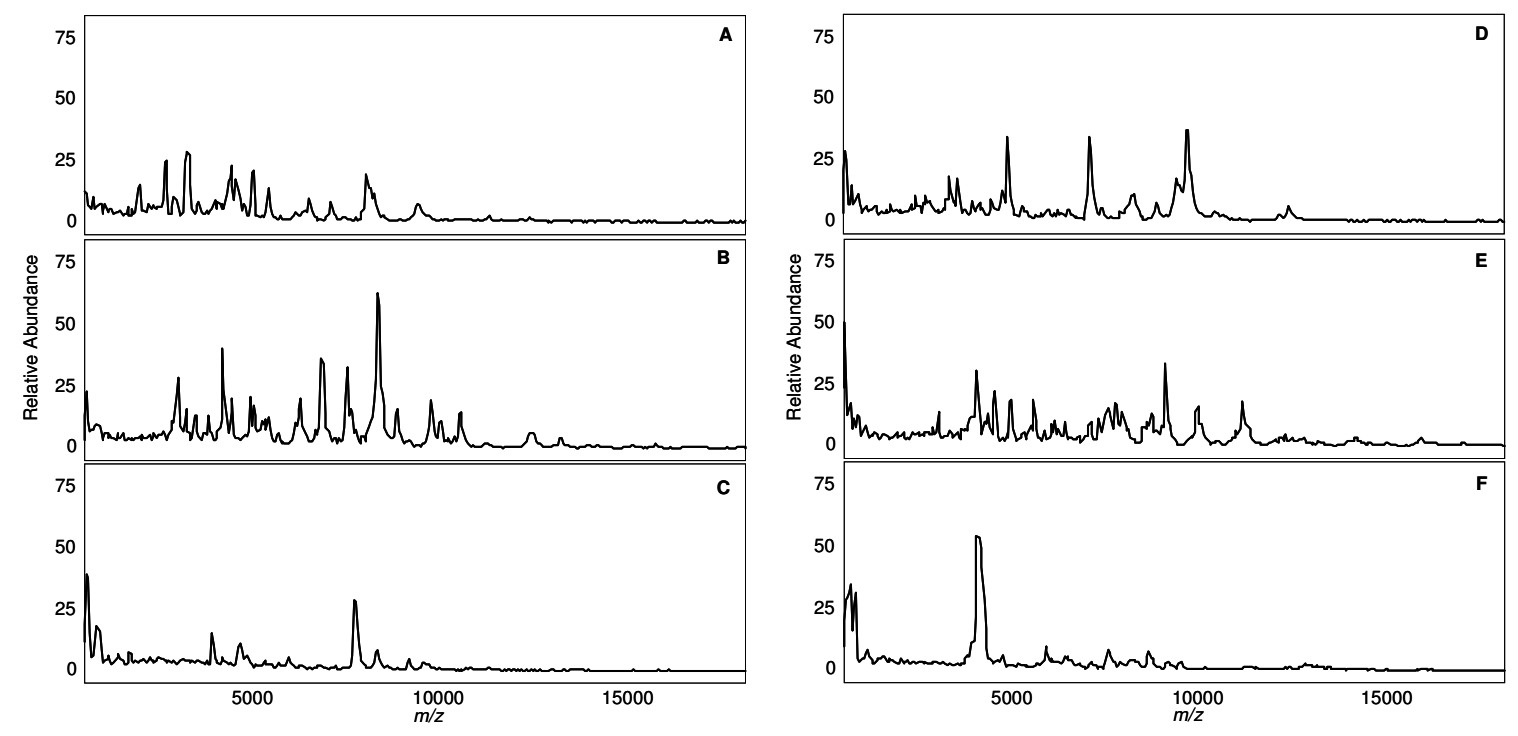

Figure 2: MALDI-TOF MS fingerprint of A) Eurotium amstelodami, B) Penicillium chrysogenum, C) Aspergillus versicolor, D) Paecilomyces variotii, E) Curvularia lunata, and F) Chaetomium globosum.

Despite the utility of MS to differentiate between these monilaceous fungi, several darkly pigmented fungi produced spectra with a paucity of peaks, and therefore prevented subsequent discriminate differentiation (Figure 3). 

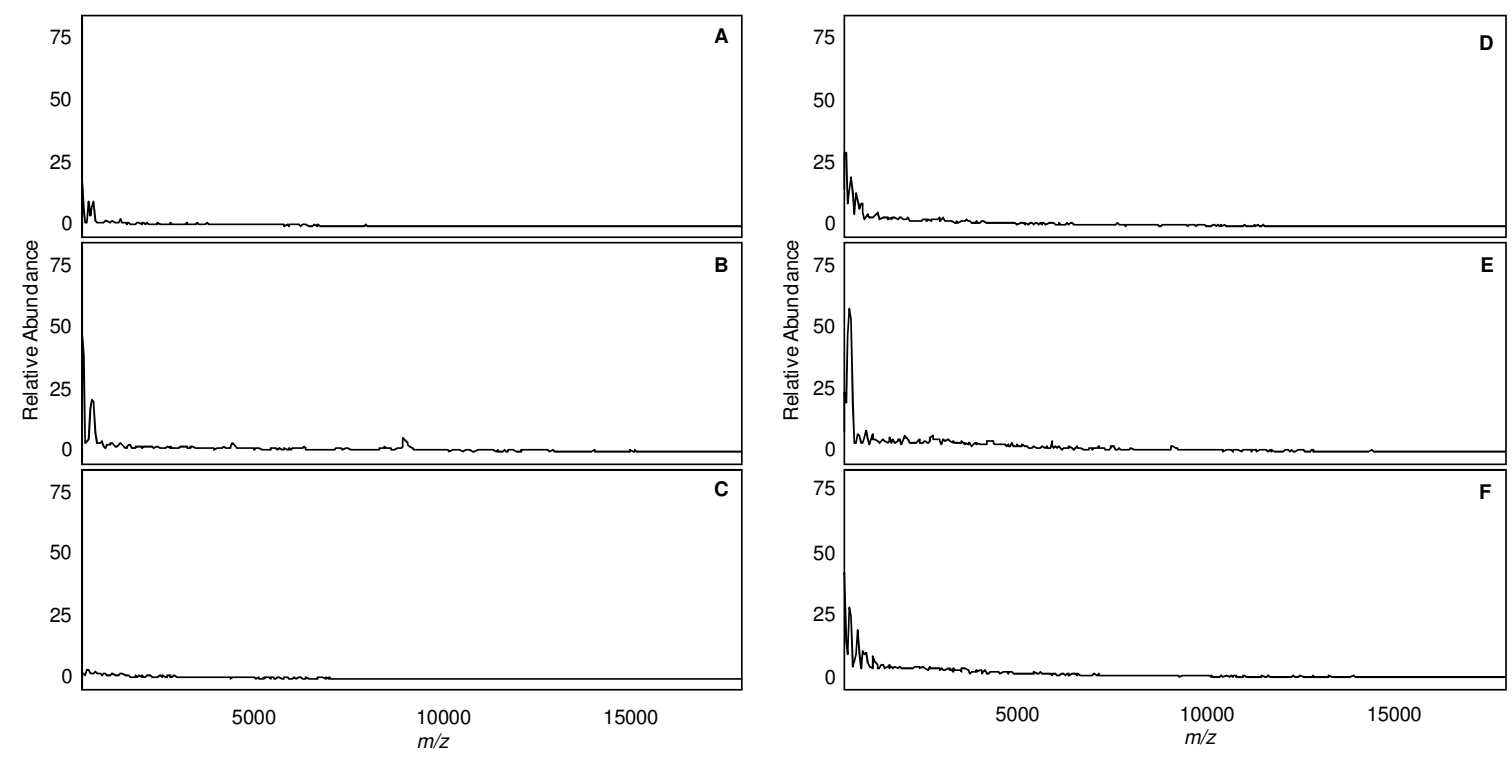

Figure 3: MALDI-TOF mass spectra of darkly pigmented fungi. A) Alternaria brassicola, B) Alternaria alternata, C) Epicoccum nigrum, D) Stachybotrys chartarum, E) Pithomyces chartarum, and F) Exserohilum rostratum.

As darkly pigmented fungi are common indoor contaminants that have been implicated in the induction and exacerbation of conditions such as asthma and allergies, it was important to obtain a MALDI-TOF MS fingerprint of these fungi to demonstrate the utility of this discrimination methodology. It was hypothesized that the presence of fungal pigments, such as melanin, in darkly pigmented fungi is preventing ionization of the analytes within the sample preparation by an unknown mechanism.

\section{Synthetic Melanin Suppression of MALDI}

In order to determine if melanin is interfering with the MALDI-TOF MS methodology, different concentrations $(0.001 \mathrm{mg} / \mathrm{mL}-20 \mathrm{mg} / \mathrm{mL})$ of commercially available synthetic melanin were spiked with human serum albumin (HSA) and then analyzed via MALDI-TOF MS. The molar concentration of 
melanin in these solutions cannot be determined from the mass of suspended melanin because melanin is a highly disperse polymer, only slightly soluble in the extraction solution, ACN/4\% TFA. The MALDITOF mass spectrum of control HSA yields a distribution of peaks corresponding to charge states from $[\mathrm{M}+\mathrm{H}]^{+}$to $[\mathrm{M}+6 \mathrm{H}]^{6+}$ (Figure $4 \mathrm{~A}$ ). Addition of synthetic melanin to the sample deposit results in a decrease in peak height in a concentration dependent manner, with the most concentrated melanin samples (> $5 \mathrm{mg} / \mathrm{mL}$ ) completely suppressing HSA peak formation. A concentration of synthetic melanin of $0.001 \mathrm{mg} / \mathrm{ml}$ spiked with HSA resulted in a $16 \%$ decrease (Figure 12).
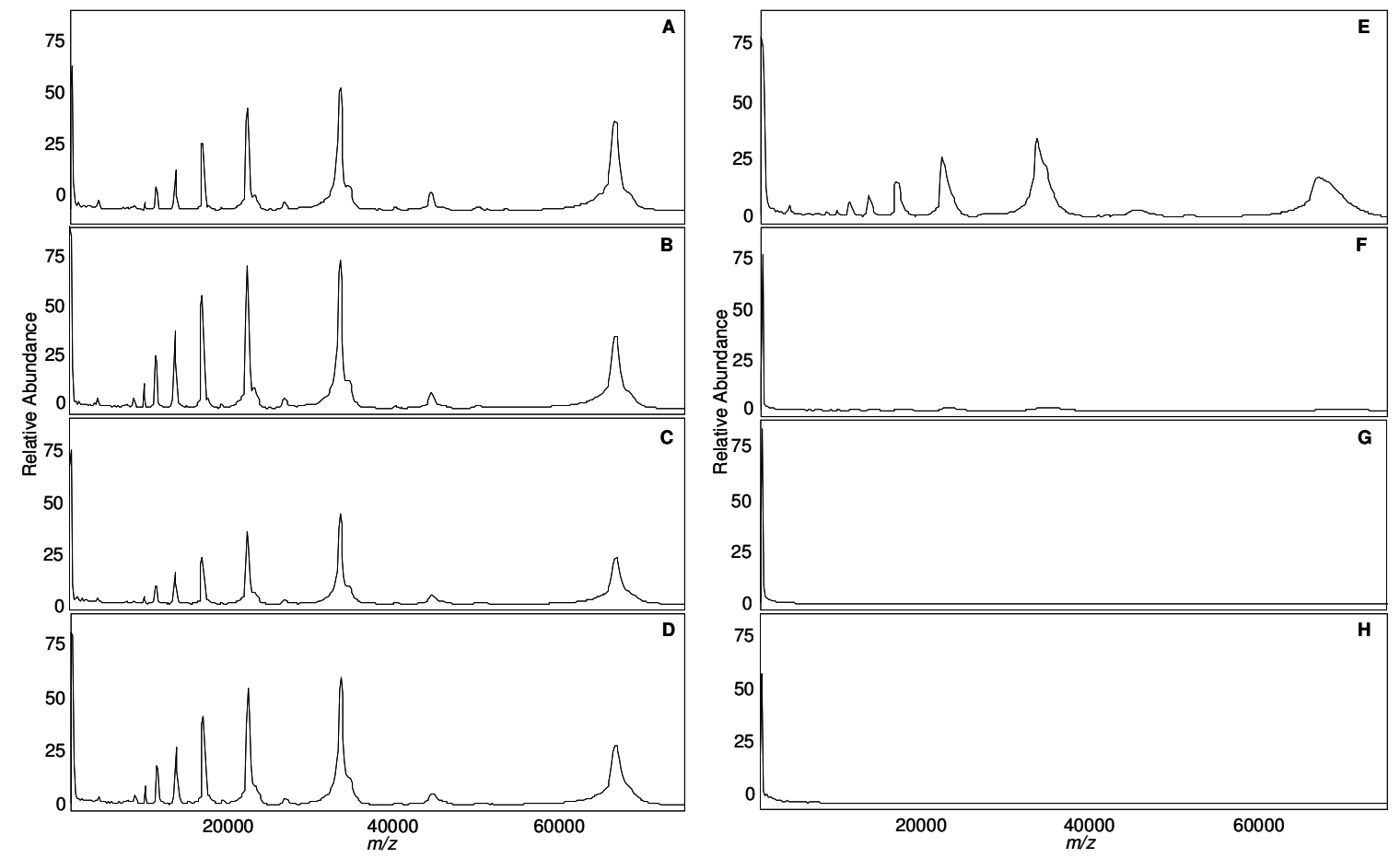

Figure 4: MALDI-TOF mass spectra of human serum albumin spiked with synthetic melanin. A) $2 X$ HSA only, B) $0.001 \mathrm{mg} / \mathrm{ml}$ synthetic melanin $+H S A, C) 0.01 \mathrm{mg} / \mathrm{ml}$ synthetic melanin $+\mathrm{HSA}, \mathrm{D}) 0.1 \mathrm{mg} / \mathrm{ml}$ synthetic melanin + HSA, E)1 mg/ml synthetic melanin + HSA, F) $5 \mathrm{mg} / \mathrm{ml}$ synthetic melanin + HSA, G) $10 \mathrm{mg} / \mathrm{ml}$ synthetic melanin $+\mathrm{HSA}$, and $H) 20 \mathrm{mg} / \mathrm{ml}$ synthetic melanin $+\mathrm{HSA}$. 


\section{Characterization of Isolated Fungal Pigments}

Because the synthetic melanin examined in the preceding experiments is not chemically identical to that obtained from fungal sources, fungal pigments were isolated from three different species of Aspergillus to determine whether these naturally occurring fungal pigments would also suppress analyte $[\mathrm{M}+\mathrm{H}]^{+}$ion formation in MALDI-TOF mass spectra. Specifically, fungal pigments from Aspergillus niger (a black fungus), A. fumigatus (green), and A. terreus (brown) were extracted and characterized prior to MALDI-TOF MS analysis. These pigments were chosen to represent the common phenotypical colors associated with most fungi, and also because of the ability to fingerprint the lighter pigmented fungal samples from A. terreus and A. fumigatus. Figure 5 shows distinguishable spectra derived from $A$. terreus and A. fumigatus; however, samples of A. niger produced spectra that lacked peaks.

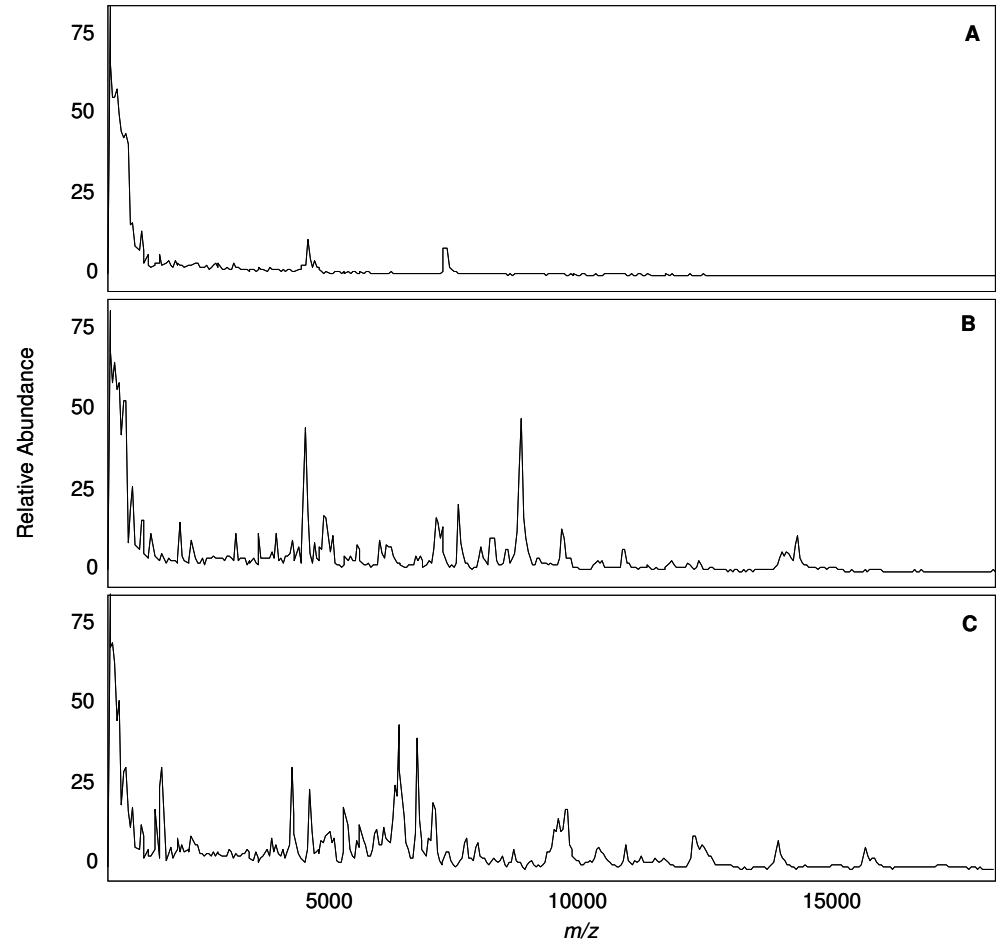

Figure 5 : MALDI-TOF MS fingerprint of fungi using protocol in Hettick et al. 2008 (64, 65). A) A. niger, B) A. fumigatus, and C) A. terreus. 
The pigment preparations were first analyzed and visualized using field emission scanning electron microscopy (FESEM). FESEM analysis suggests that fungal pigments isolated from A. niger and A. fumigatus maintain the ultrastructural architecture of the fungal spores following melanin extraction (Figure 6A-B). This observation is consistent with previous reports of pigments isolated from other fungal species, including Cryptococcus neoformans and the term "melanin ghosts" has been coined (91, 93). In contrast, pigments extracted from A. terreus were structurally similar to the commercially available synthetic melanin (Figure 6 C-D).

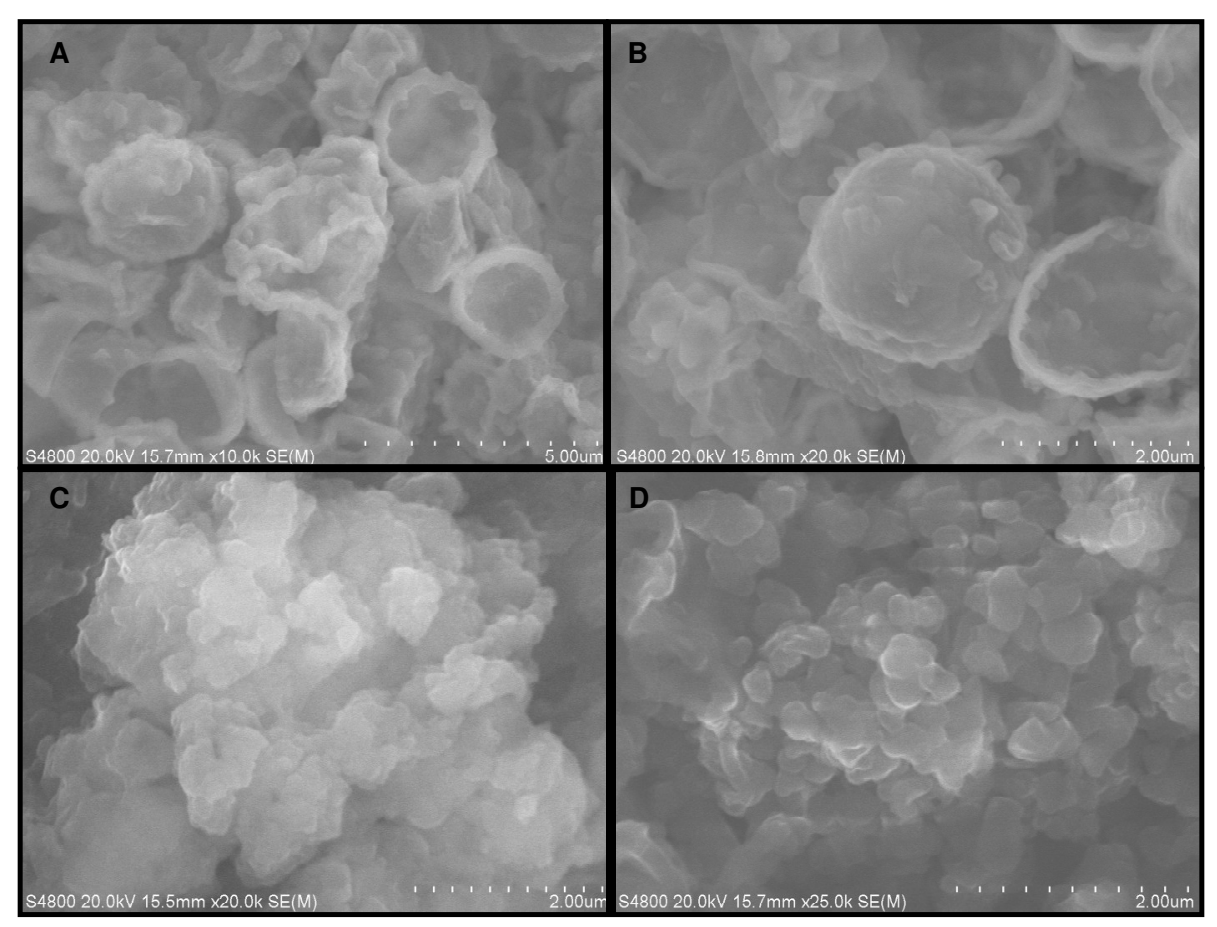

Figure 6: FESEM images of pigment extracts or synthetic melanin. A) A. niger pigment extract, B) A. fumigatus pigment extract, C) A. terreus pigment extract, and D) synthetic melanin.

In addition to the FESEM, elemental analysis was also conducted to determine the chemical make-up of each sample because different types of melanins contain different concentrations of carbon, nitrogen, hydrogen, and in some cases sulfur. There were no differences between each sample in the 
chemical components, as each pigment contained high levels of carbon, oxygen, hydrogen, and sulfur, while none contained nitrogen (data not shown).

Gas chromatography/mass spectrometry (GC/MS) was also conducted on each sample to determine the presence of different functional groups within each sample. GC/MS analysis indicated the presence of long chain fatty acids and aliphatic compounds in each fungal pigment preparation which was not present in synthetic melanin (Figure 7A), most likely due to the presence of cell wall components within the samples (Figure 7B). These results are consistent with DHN-melanins, which are known to be extensively bound to cell wall/structural components of the A. fumigatus spore.
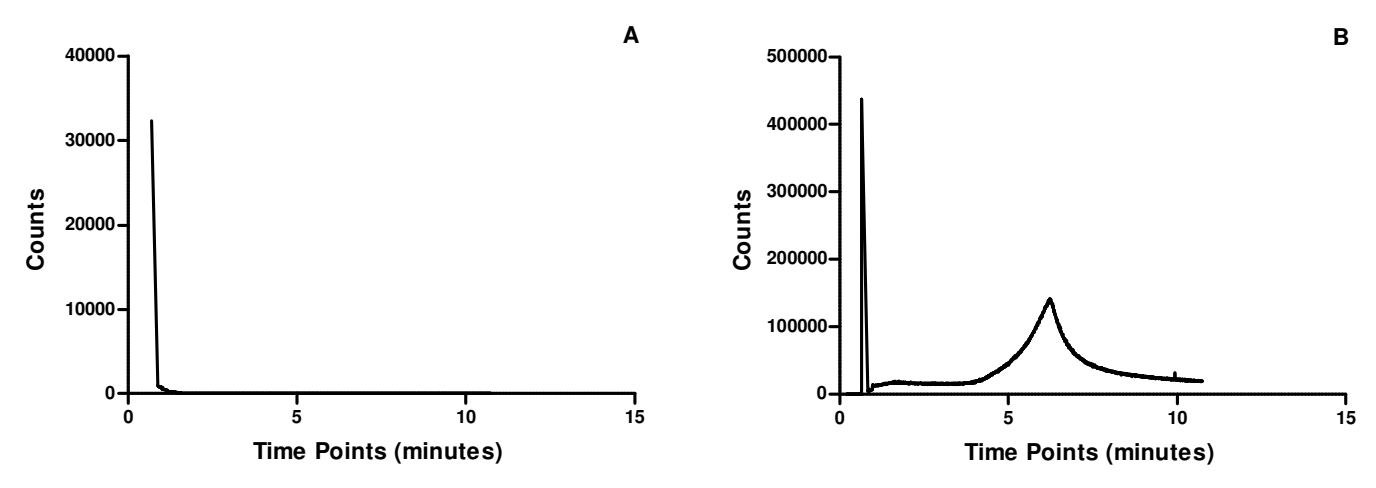

Figure 7: GC/MS spectra of A) Synthetic melanin or B) A. niger pigment extract. The broad peak present in B indicates the presence of fatty acid chains and aliphatic compounds.

In addition, UV spectroscopy was done on each pigment extract. Each pigment $(1 \mathrm{mg} / \mathrm{mL})$ exhibited strong, non-zero, absorption in the $200-800 \mathrm{~nm}$ range when analyzed by UV/VIS spectroscopy, and absorption approached 1.0 at $337-\mathrm{nm}$, the emission wavelength of the nitrogen laser used for MALDI-TOF MS. An example of A. niger pigment extract absorption when solubilized in 50/50 hydrofluoric acid/nitric acid is shown in Figure 8. Pigments from other fungi yielded similar spectra. 


\section{UV/Vis A. niger Pigment Extract Spectrum}

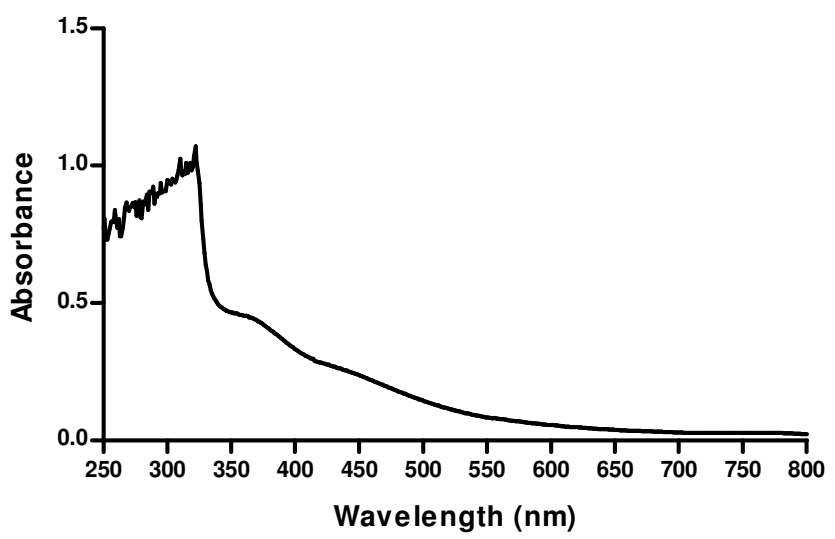

Figure 8: UV/Vis spectrum of $1 \mathrm{mg} / \mathrm{mL}$ A. niger in 50/50 hydrofluoric acid/nitric acid.

This observation is consistent with previous studies that demonstrated melanin to have photoprotective properties in fungi, absorbing UV radiation and dissipating energy as heat via rapid internal conversion (84-86). Therefore, it is possible that there is competition between the fungal pigment and the analytes within the sample, whereby the pigments are absorbing photons, preventing ionization of the analytes and subsequently inhibiting the spectrum formation.

\section{Suppression of MALDI-TOF MS by Isolated Fungal Pigments}

The isolated pigments from each of these fungi were analyzed for protein content before being spiked into HSA, none was present. In these experiments, a concentration dependent inhibition of the $[\mathrm{M}+\mathrm{H}]^{+}$ion peak height was observed with each fungal pigment extract (Figure 12), similar to that seen with synthetic melanin. The most concentrated sample $(20 \mathrm{mg} / \mathrm{mL})$ of $A$. niger pigment reduced the 66 $\mathrm{m} / \mathrm{z}$ peak abundance by $96 \%$, while $1 \mathrm{mg} / \mathrm{mL}$ of pigment reduced the peak height by $8 \%$ (Figures 9 and 12). A. fumigatus pigment (which did not prevent the acquisition of a unique fingerprint of the fungal sample) reduced the peak height by $89 \%$ at $20 \mathrm{mg} / \mathrm{mL}$, while the $0.001 \mathrm{mg} / \mathrm{mL}$ sample reduced the peak height by $10 \%$ (Figures 10 and 12). Interestingly, the pigment isolated from A. terreus reduced the HSA peak height by $96 \%$ at $20 \mathrm{mg} / \mathrm{mL}$ and by $8 \%$ at $1 \mathrm{mg} / \mathrm{mL}$ (Figures 11 and 12), which was unexpected 
because A. terreus is light brown in color when grown on malt extract agar and the fungi itself produces a spectrum that contains multiple peaks of high abundance as shown in Figure 5.
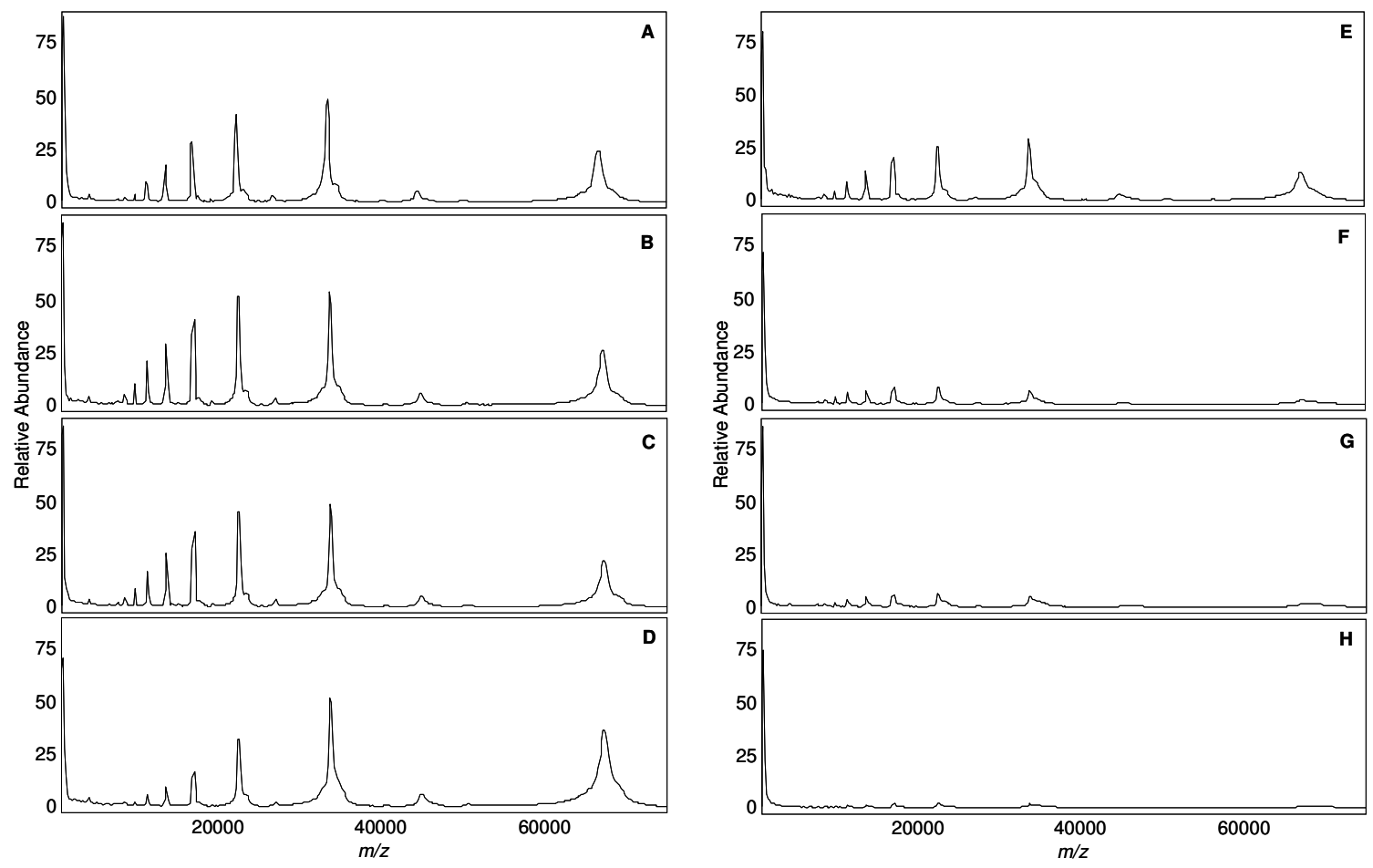

Figure 9: MALDI-TOF mass spectra of human serum albumin spiked A. niger pigment preparations. A) 2X HSA only, B) $0.001 \mathrm{mg} / \mathrm{ml}$ A. niger pigment $+H S A, C) 0.01 \mathrm{mg} / \mathrm{ml}$ A. niger pigment + HSA, D) 0.1 mg/ml A. niger pigment + HSA, E) $1 \mathrm{mg} / \mathrm{ml}$ A. niger pigment + HSA, F) $5 \mathrm{mg} / \mathrm{ml}$ A. niger pigment + HSA, G) $10 \mathrm{mg} / \mathrm{ml}$ A. niger pigment + HSA, and H) $20 \mathrm{mg} / \mathrm{ml}$ A. niger pigment + HSA. 

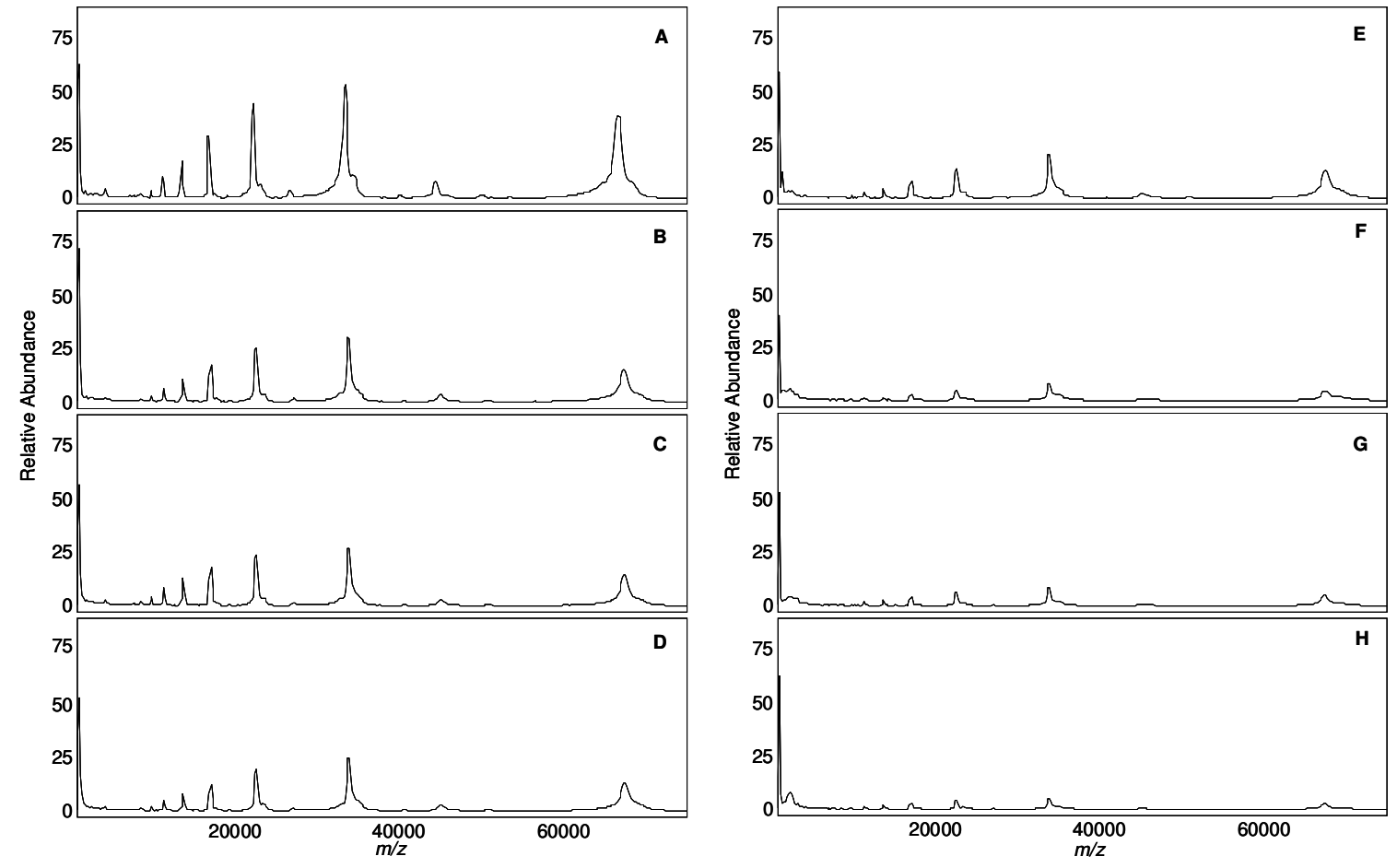

Figure 10: MALDI-TOF mass spectra of human serum albumin spiked with A. fumigatus pigment preparations. A) $2 X \mathrm{HSA}$ only, B) $0.001 \mathrm{mg} / \mathrm{ml}$ A. fumigatus pigment $+\mathrm{HSA}$, C) $0.01 \mathrm{mg} / \mathrm{ml} \mathrm{A}$. fumigatus pigment $+H S A, D) 0.1 \mathrm{mg} / \mathrm{ml}$ A. fumigatus pigment $+H S A, E) 1 \mathrm{mg} / \mathrm{ml}$ A. fumigatus pigment $+H S A, F)$ $5 \mathrm{mg} / \mathrm{ml}$ A. fumigatus pigment $+\mathrm{HSA}, \mathrm{G}) 10 \mathrm{mg} / \mathrm{ml}$ A. fumigatus pigment $+\mathrm{HSA}$, and $\mathrm{H}) 20 \mathrm{mg} / \mathrm{ml} \mathrm{A}$. fumigatus pigment $+H S A$. 

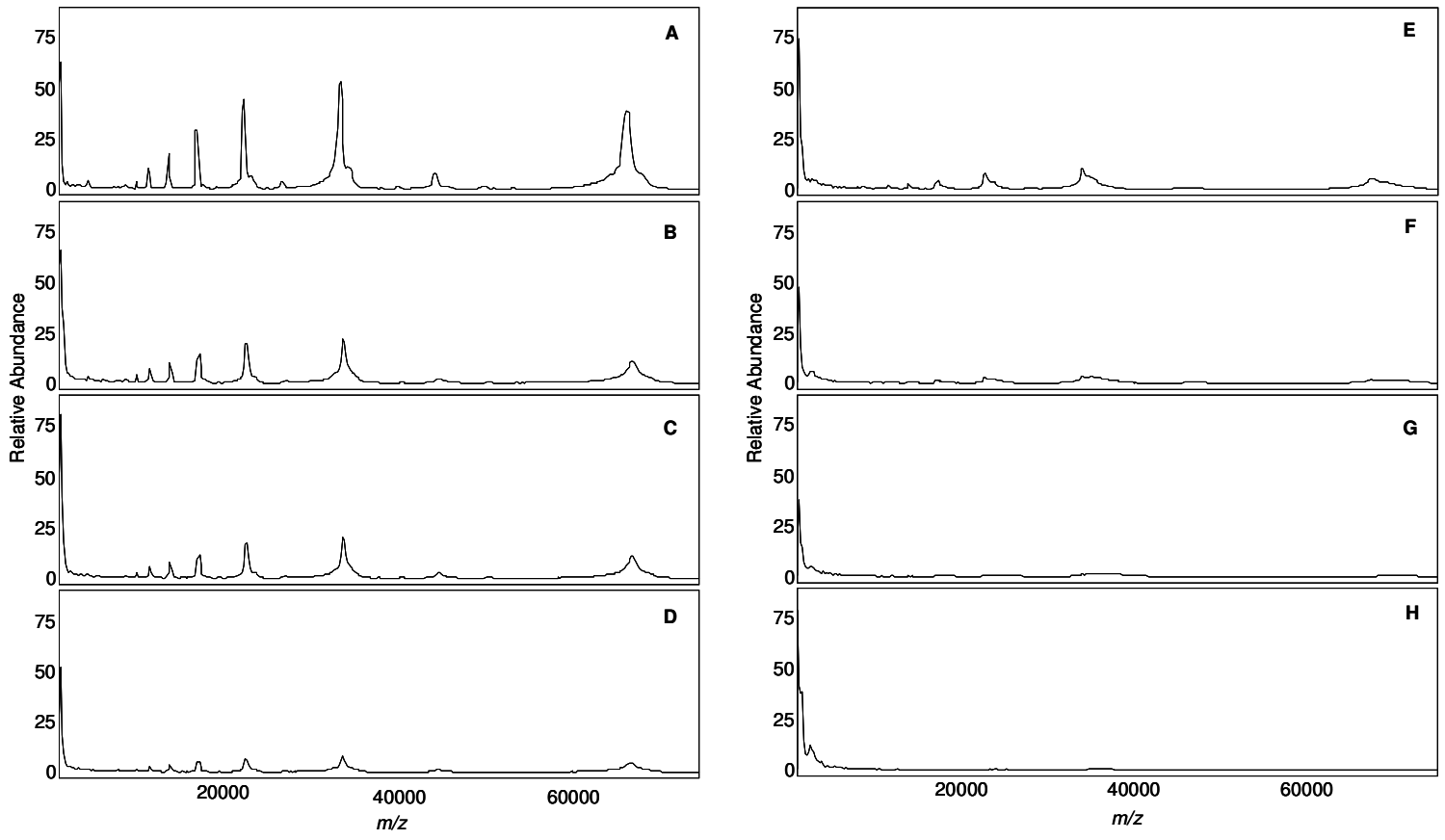

Figure 11: MALDI-TOF mass spectra of human serum albumin spiked with A. terreus pigment preparations. A) $2 X \mathrm{HSA}$ only, B) $0.001 \mathrm{mg} / \mathrm{ml}$ A. terreus pigment $+\mathrm{HSA}$, C) $0.01 \mathrm{mg} / \mathrm{ml} \mathrm{A}$. terreus pigment $+H S A, D) 0.1 \mathrm{mg} / \mathrm{ml}$ A. terreus pigment $+H S A, E) 1 \mathrm{mg} / \mathrm{ml}$ A. terreus pigment $+H S A, F) 5$ $\mathrm{mg} / \mathrm{ml}$ A. terreus pigment $+\mathrm{HSA}$, G) $10 \mathrm{mg} / \mathrm{ml}$ A. terreus pigment $+\mathrm{HSA}$, and H) $20 \mathrm{mg} / \mathrm{ml} \mathrm{A}$. terreus pigment + HSA . 


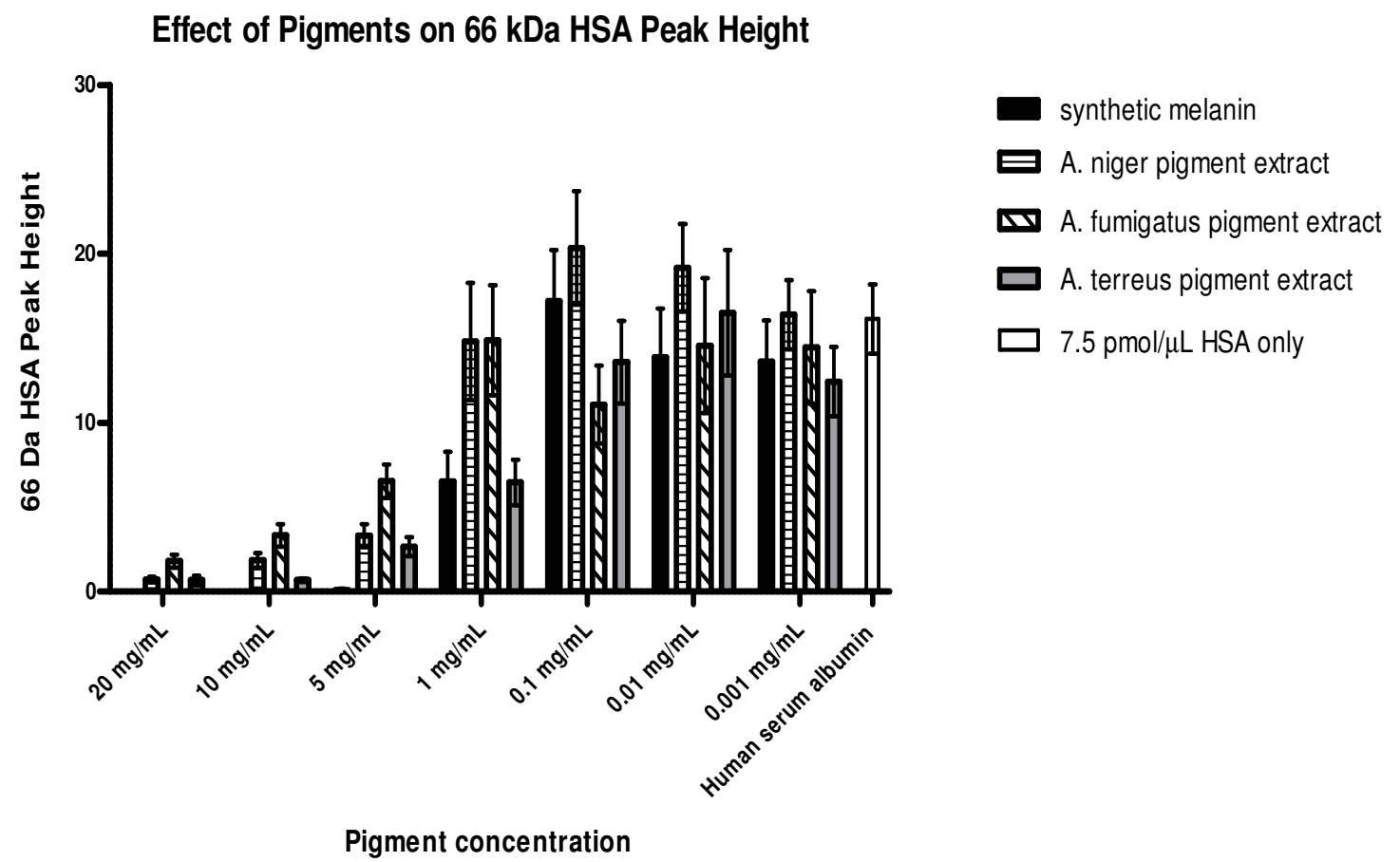

Figure 12: Effect of fungal pigment suppression of $66 \mathrm{~m} / \mathrm{z}$ HSA peak height. Data values are reported as the mean \pm the standard error of measure.

Because melanin is poorly soluble under MALDI sample deposit conditions (e.g. 50/50 ACN/4\% TFA), the pigment samples analyzed in these experiments were suspensions, not homogenous solutions. Further, MALDI sample deposits are often heterogeneous, leading to poor shot-to-shot and deposit-todeposit reproducibility $(94,95)$. Thus, sample deposits used for MALDI-TOF MS analysis which were visualized via light microscopy at $4 \mathrm{X}$ magnification and appeared heterogeneous, with regions of high and low pigment levels across the sample deposit (Figure 13). 


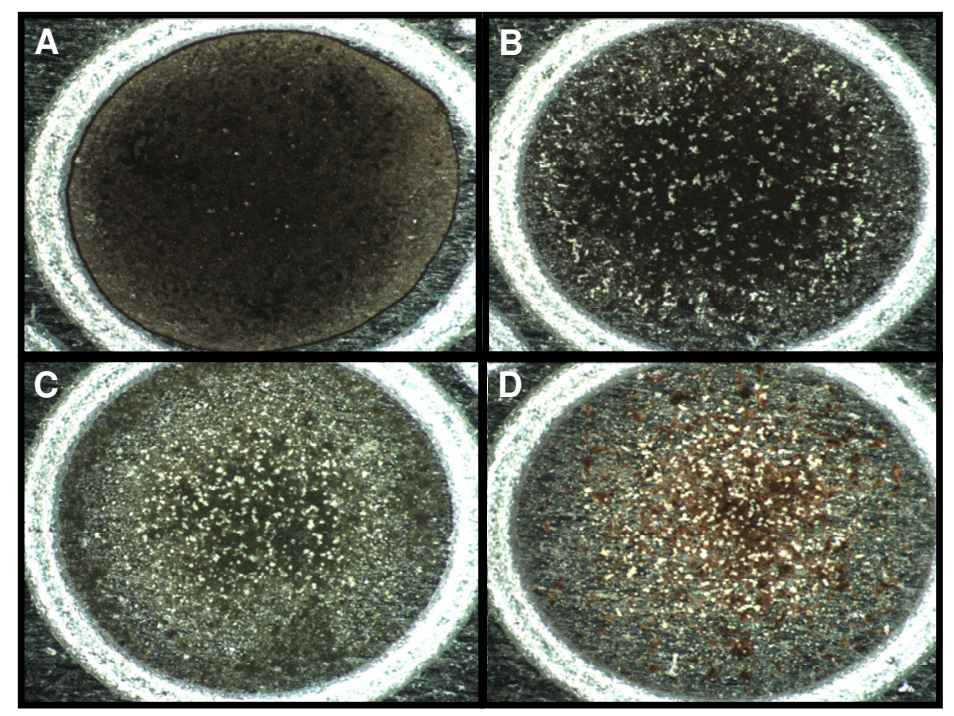

Figure 13: Light microscopy images of sample spots on a MALDI target chip of A) synthetic melanin, B) A. niger pigment extract, C) A. fumigatus pigment extract, and D) A. terreus pigment extract under $4 X$ magnification. Equal concentration of each pigment were mixed with matrix and spotted.

This observation may account for the HSA peak height inhibition in those samples. Since the synthetic melanin was present more homogenously throughout the spot, as opposed to the aggregated pigments from A. niger and A. fumigatus, this presents a greater likelihood that the MALDI UV-laser would fire on a spot where pigment was more concentrated. To examine the effect of this heterogeneity on the acquired MALDI-TOF mass spectrum, a sample of $10 \mathrm{pmol} / \mu \mathrm{L}$ of angiotensin II was spiked with an equal volume of $20 \mathrm{mg} / \mathrm{mL}$ A. fumigatus pigment extract and analyzed by high resolution MALDITOF MS (Figure 14). On this instrument, the sample deposit is imaged by a camera and the exact spatial location of the MALDI laser output can be guided in real time. Spectra were acquired from regions of high and low A. fumigatus pigment concentration (empirically determined by black coloration). Direct suppression of the angiotensin II $[\mathrm{M}+\mathrm{H}]^{+}$ion $(1046 \mathrm{~m} / \mathrm{z})$ signal in regions of high pigment concentration is observed, whereas a strong angiotensin II $[\mathrm{M}+\mathrm{H}]^{+}$ion signal is observed from regions of low pigment concentration. 


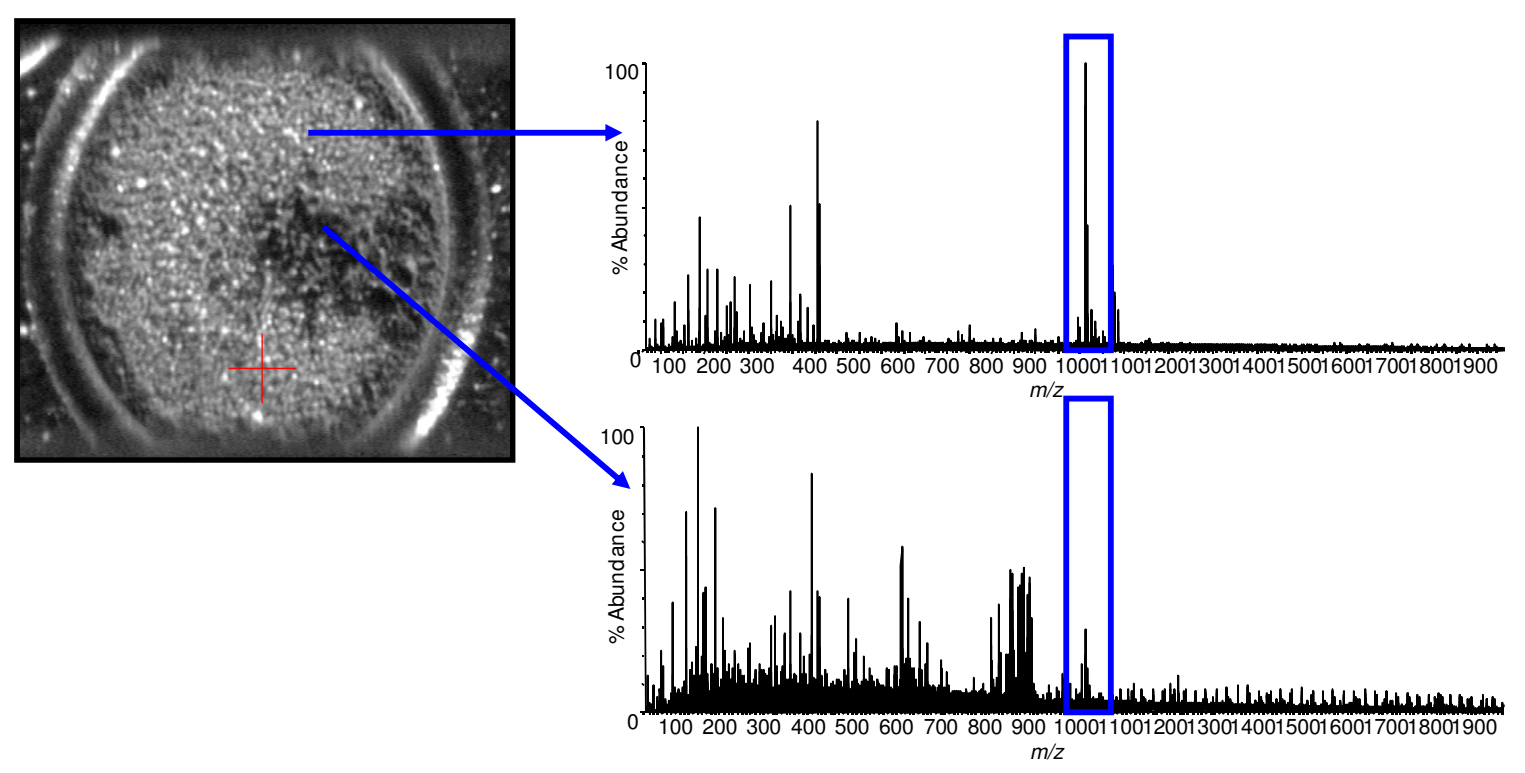

Figure 14: MALDI Q-TOF spectra of A. fumigatus pigment extract spiked with $0.5 \mathrm{mg} / \mathrm{ml}$ angiotensin II (singly charged peak around $1000 \mathrm{~m} / \mathrm{z}$ ) spike with A. fumigatus pigment extract. Top spectrum is taken from an area with no visible pigment. Bottom spectrum is taken from a spot with a high concentration of pigment extract.

\section{Fungal Culture Conditions Effect Melanin Production}

The preceding experiments clearly demonstrate that melanin pigments produced by fungi are at least, in part, responsible for the observed suppression of MALDI ionization in fingerprint mass spectra from darkly pigmented fungi. Thus, it was further hypothesized that if melanin production could be blocked in darkly pigmented fungi, problematic species, such as A. niger, would yield fingerprint mass spectra similar to their unmelanized relatives. Many fungi, when grown for short durations in liquid culture, do not produce pigments $(29,81)$. Thus, melanin-free Aspergillus cultures were produced by growing each of the three species in liquid media. Unique MALDI-TOF MS fingerprint spectra were acquired from each fungus grown in liquid culture, including A. niger (Figure 15). 


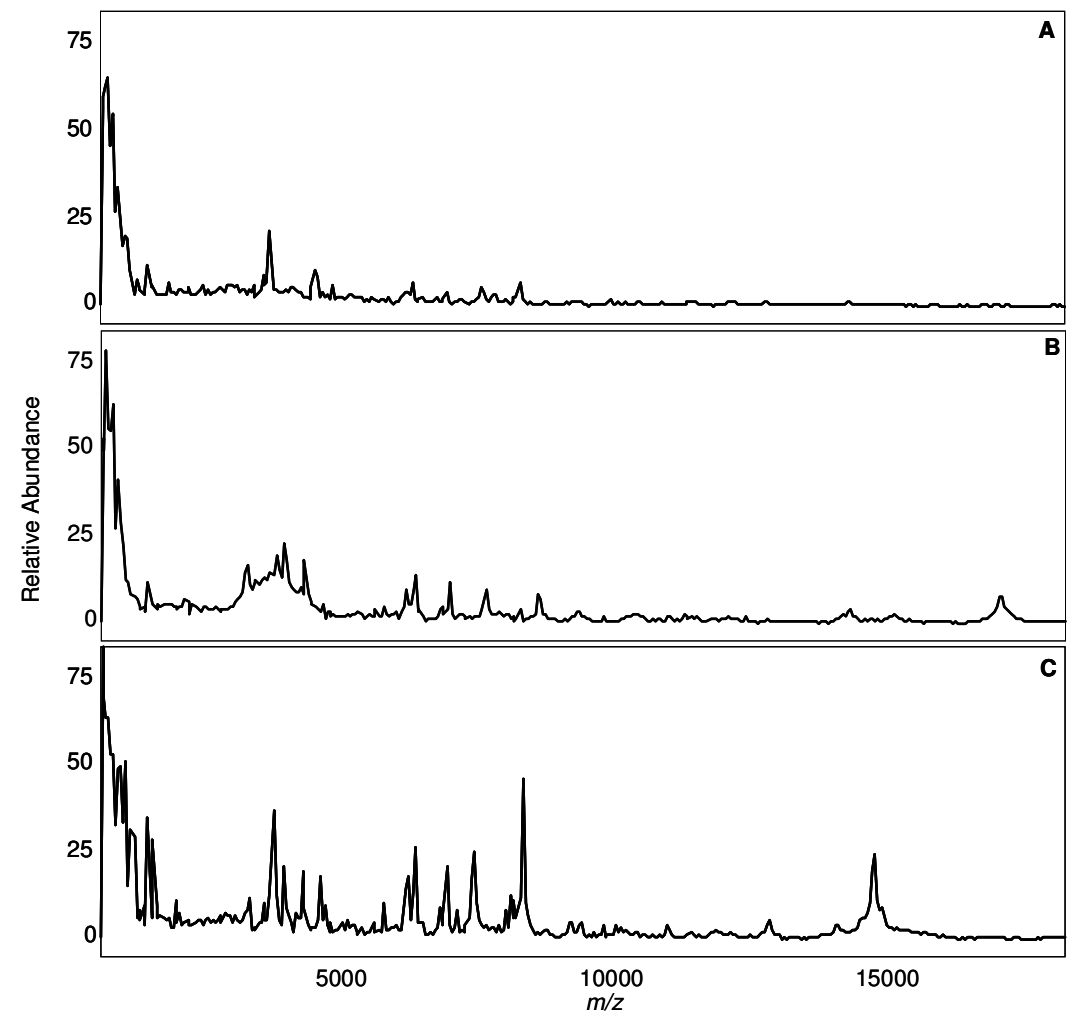

Figure 15: MALDI-TOF mass spectra of cultures grown under submerged conditions in malt extract broth. A) A. niger, B) A. fumigatus, and C) A. terreus.

The MALDI-TOF mass spectrum for each species grown under submerged conditions contains sufficient information to allow these species to be easily discriminated. Contrast the MALDI-TOF fingerprint mass spectrum for A. niger grown under submerged conditions (Figure 15A) with that of $A$. niger grown on MEA (Figure 5A). Specifically, five significant peaks were identified, and the replicate cultures grouped together and were correctly identified with only a $4 \%$ error rate. Two canonical functions were used to properly discriminate and group these fungi based predominantly on the peak intensity (Figure 16). 


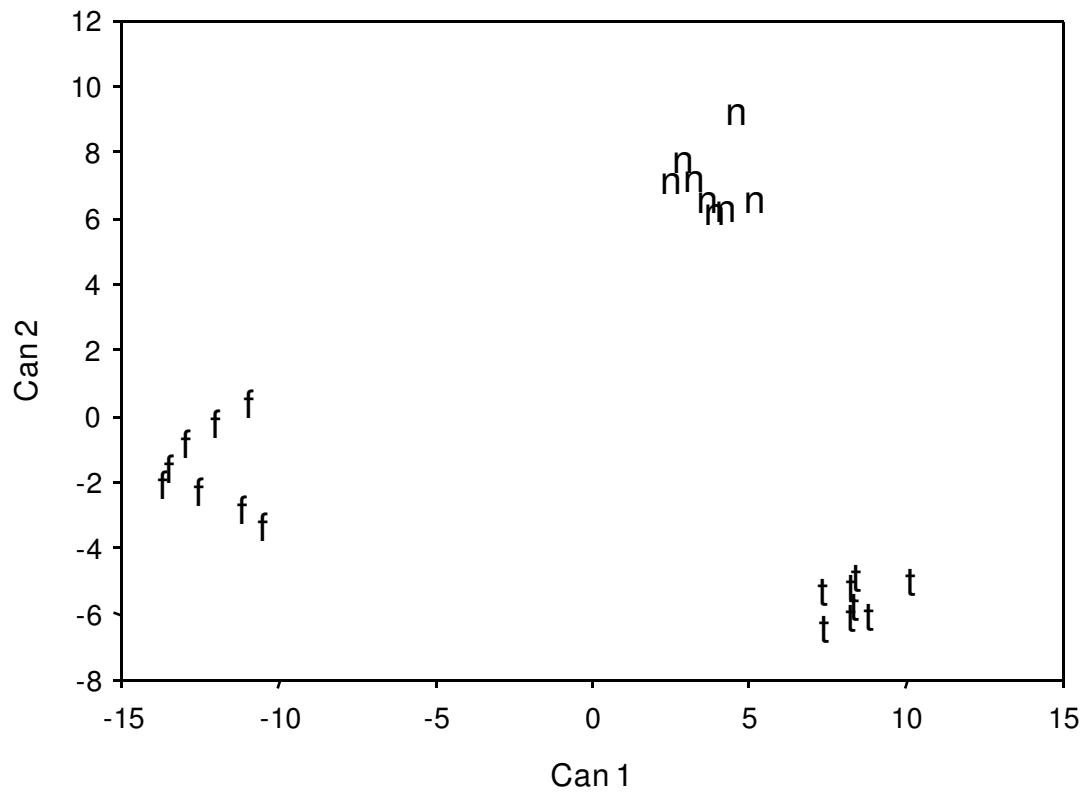

Figure 16: $2 D$ canonical distribution of fungi grown under submerged culture conditions, $n=8$ replicate A. niger extracts, $f=8$ replicate A. fumigatus extracts, and $t=8$ replicate A. terreus extracts.

\section{Inhibition of Melanin Production via Tricyclazole}

Fungi grown in submerged conditions form mycelial masses, a different growth state than sporulation, which has been used in previous studies in our laboratory $(64,65)$. Because fungi grown under submerged conditions may contain altered protein concentrations and exhibit significant changes to the fingerprint mass spectrum, it makes comparison with sporulating MEA cultures of other Aspergilli difficult. Therefore, it was necessary to grow fungi under sporulating conditions and to block melanin production to determine if MALDI-TOF MS fingerprint mass spectra could be acquired from these species. Therefore, each fungal species was grown under sporulating conditions identical to our previously published fungal mass fingerprinting methodology, but in the presence of tricyclazole. Tricyclazole is an antifungal agent that specifically inhibits DHN-melanin formation by inhibiting two reductases critical to the pentaketide DHN-melanin synthesis pathway (96). Therefore, fungi were grown on MEA containing tricyclazole, and then analyzed as above. It is important to note that A. fumigatus, when grown on agar containing tricyclazole, appeared light brown in color, similar to A. terreus, 
however, when grown on MEA only, the color of the culture is dark green. Conversely, when A. niger was grown under tricyclazole conditions, a significant color change was not noted (Figure 17).

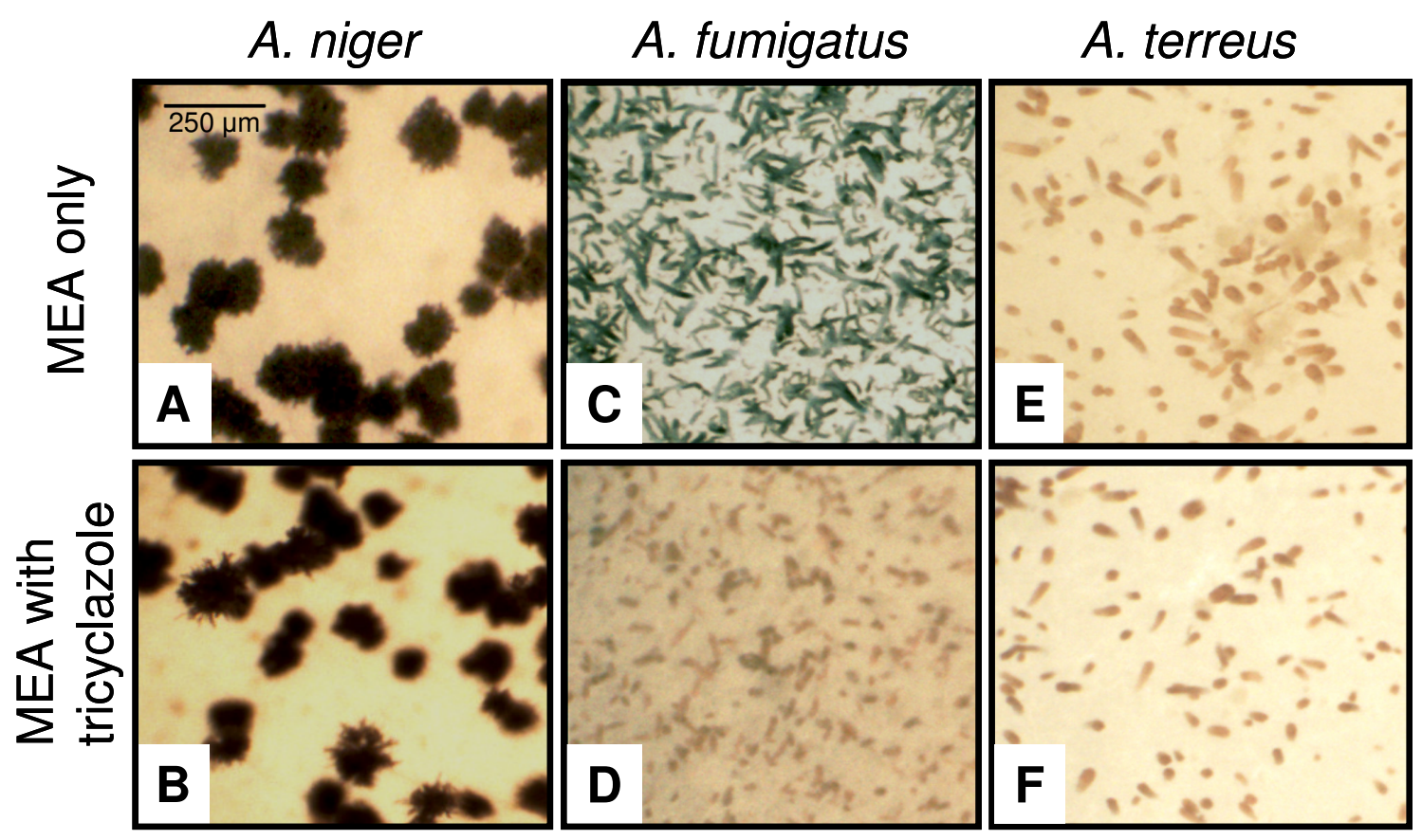

Figure 17: Appearance of cultures when grown on MEA only (top) or MEA supplemented with50 $\mu \mathrm{g} / \mathrm{mL}$ (bottom) for 7 days. A) A. niger grown on MEA, B) A. niger grown on MEA with tricyclazole, C) A. fumigatus grown on MEA, D) A. fumigatus grown on MEA with tricyclazole, E) A. terreus grown on $M E A$, and F) A. terreus grown on MEA with tricyclazole.

In general, spectra obtained from cultures grown on agar containing tricyclazole for 14 days contained peaks that were of higher abundance than the spectra obtained from samples grown on MEA only, despite the difference in phenotypical color not being visibly altered in A. niger or A. terreus. This was evident for cultures of $A$. niger, where peaks were rarely discernable from the background noise of the spectra when the fungus was grown on MEA only, however spectra from A. niger grown on agar supplemented with tricyclazole five dominant peaks were present, as shown in Figure 18. 


\section{Days}

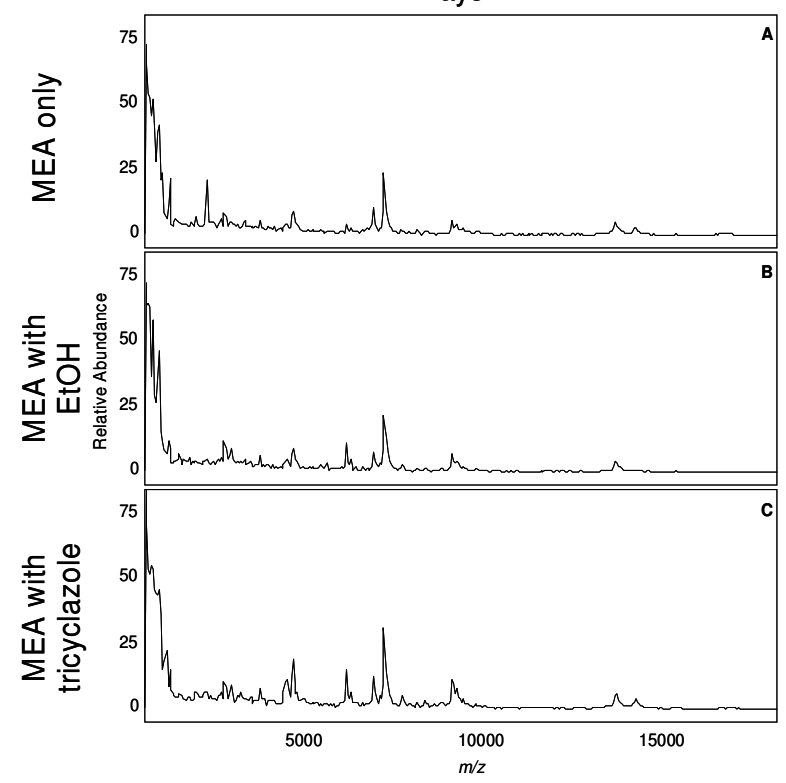

14 Days

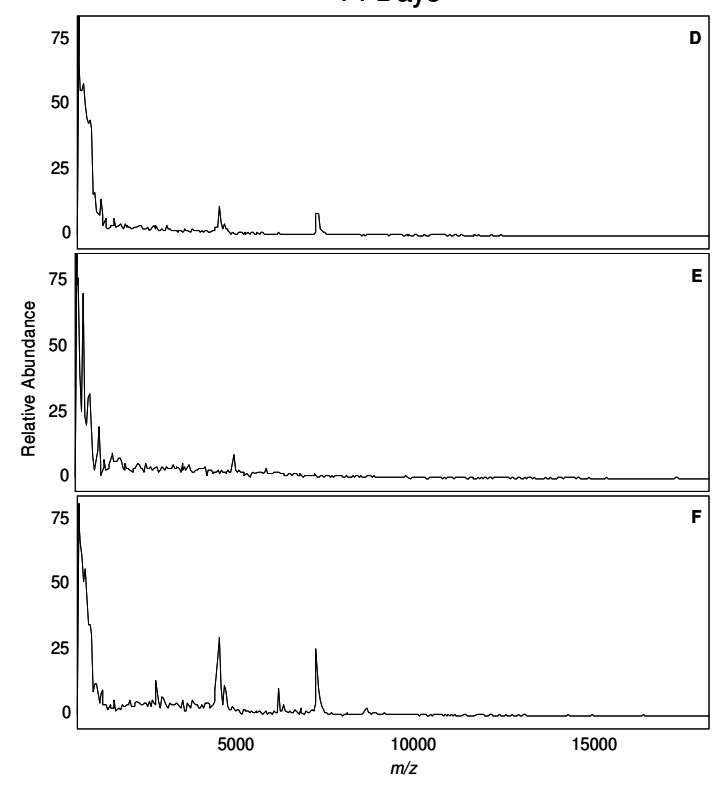

Figure 18: MALDI-TOF mass spectra of A. niger cultures grown for 7 days (left) or 14 days (right). A) 7 days A. niger $M E A, B) 7$ days A. niger $M E A+1 \%$ EtOH, C) 7 days A. niger $M E A+1 \%$ EtOH +50 $\mu \mathrm{g} / \mathrm{ml}$ tricyclazole, D) 14 days A. niger MEA, E) 14 days A. niger MEA $+1 \%$ EtOH, and F) 14 days A. niger $\mathrm{MEA}+1 \% \mathrm{EtOH}+50 \mu \mathrm{g} / \mathrm{ml}$ tricyclazole.

In addition, spectra from $A$. terreus and A. fumigatus grown on both MEA and MEA containing tricyclazole contained peaks of matching $\mathrm{m} / \mathrm{z}$, however, those grown in tricyclazole conditions appeared as increased peak intensity (Figures 19 and 20). Peak intensity is an important variable for the ability to canonically discriminate between the samples, as shown from the data obtained from the submerged culture identification. 

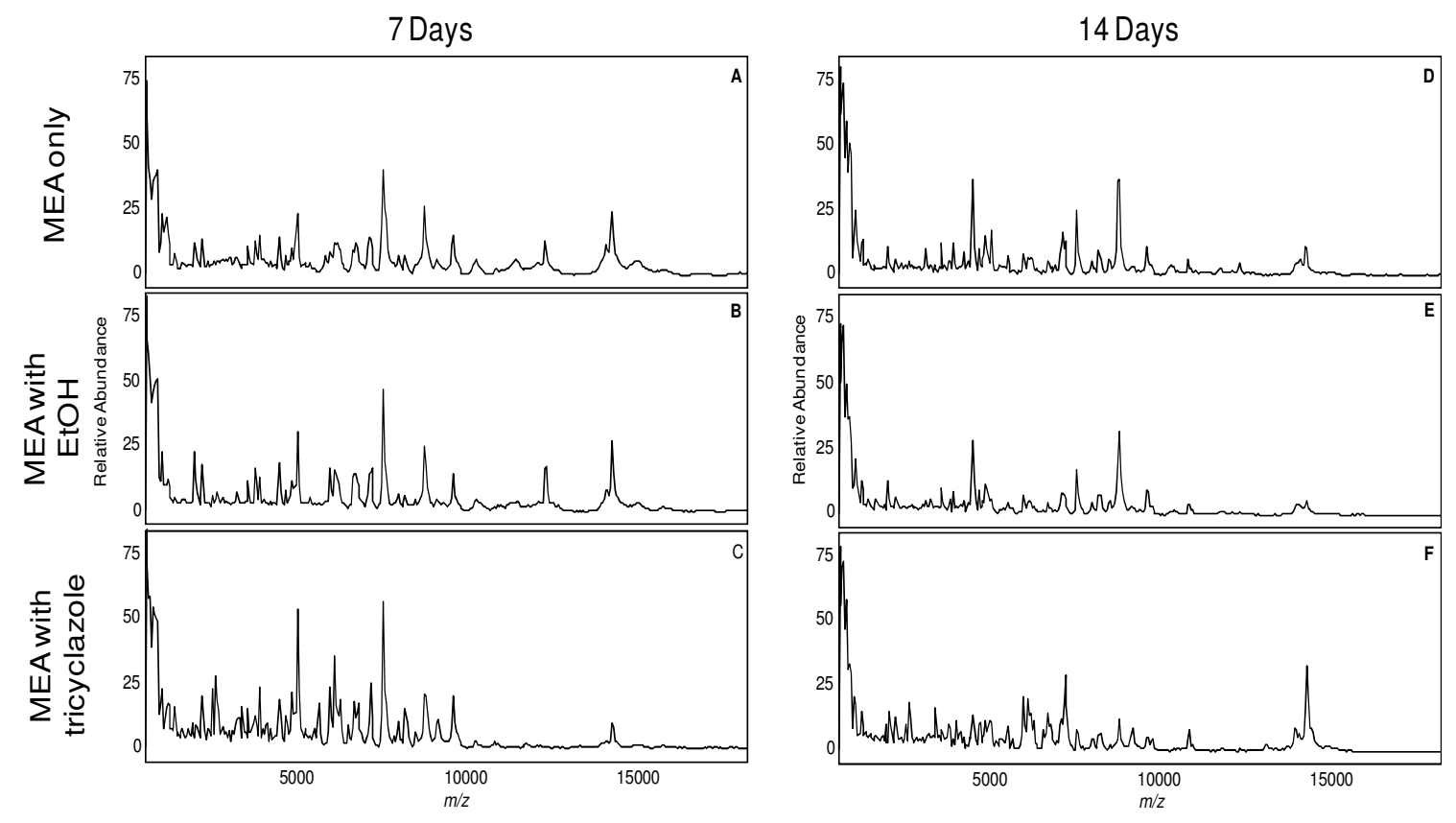

Figure 19: MALDI-TOF mass spectra of A. fumigatus cultures grown for 7 days (left) or 14 days (right). A) 7 days A. fumigatus MEA, B) 7 days A. fumigatus $M E A+1 \% E t O H, C) 7$ days A. fumigatus $M E A+$ $1 \% \mathrm{EtOH}+50 \mu \mathrm{g} / \mathrm{ml}$ tricyclazole, D) 14 days A. fumigatus MEA, E) 14 days A. fumigatus $M E A+1 \%$ EtOH, and $\mathrm{F}) \mathrm{A}$. fumigatus 14 days $\mathrm{MEA}+1 \% \mathrm{EtOH}+50 \mu \mathrm{g} / \mathrm{ml}$ tricyclazole. 

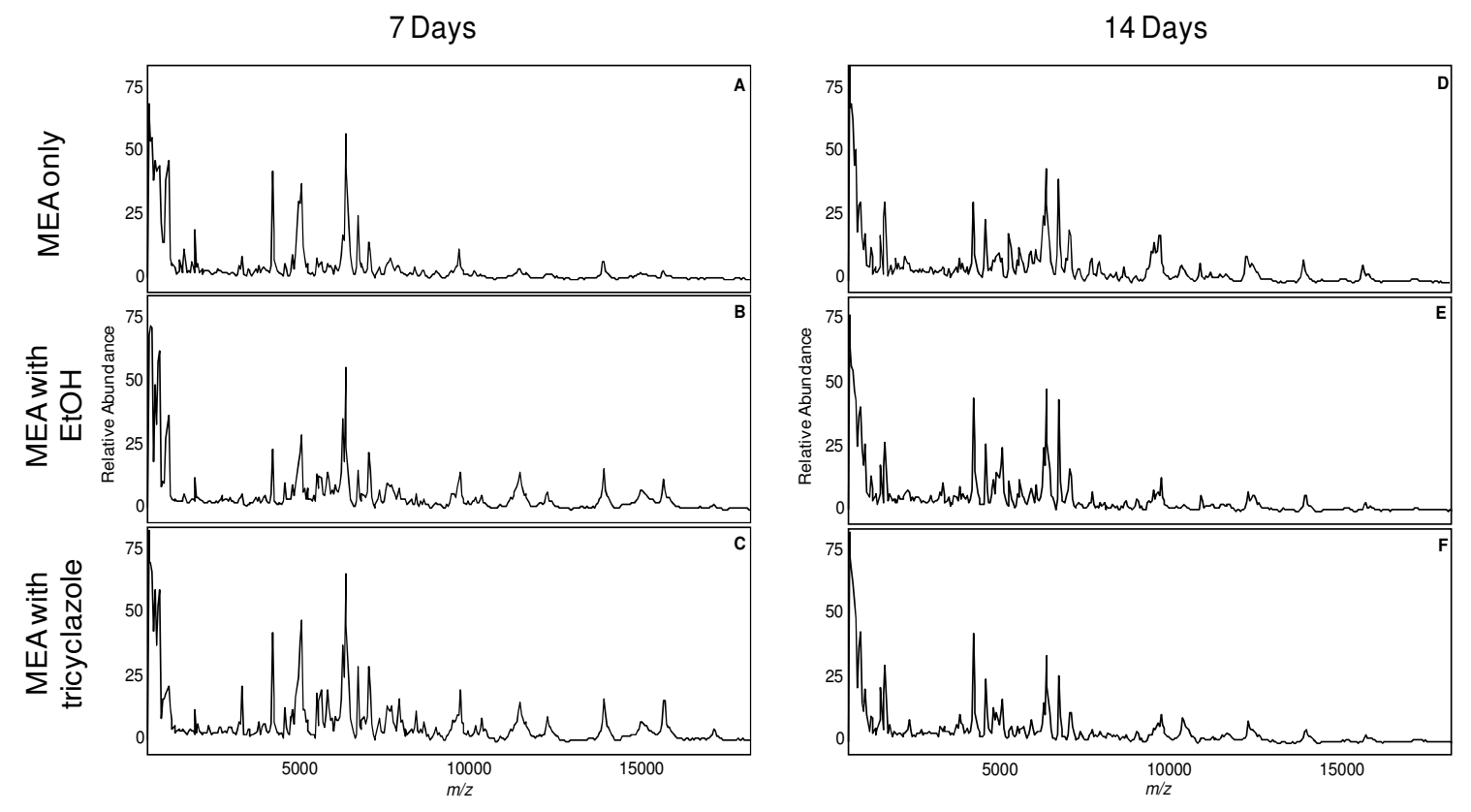

Figure 20: MALDI-TOF mass spectra of A. terreus cultures grown for 7 days (left) or 14 days (right). A) 7 days A. terreus $M E A, B) 7$ days A. terreus $M E A+1 \%$ EtOH, C) 7 days A. terreus $M E A+1 \%$ EtOH + $50 \mu \mathrm{g} / \mathrm{ml}$ tricyclazole, D) 14 days A. terreus MEA, E) 14 days A. terreus $\mathrm{MEA}+1 \% \mathrm{EtOH}$, and F) 14 days A. terreus $\mathrm{MEA}+1 \% \mathrm{EtOH}+50 \mu \mathrm{g} / \mathrm{ml}$ tricyclazole.

Another variable that was examined was culture time. It was hypothesized that the longer the cultures were grown, the more spectra inhibiting melanin would be produced and retained within the fungal spore; therefore, fungi grown for a shorter duration would contain less melanin, and thus produce a unique fingerprint with discernable peaks. In this series of experiments, cultures were grown for seven days under the same conditions as above, and analyzed as previously described. Spectra were obtained from each species from MEA only, control MEA with ethanol, and MEA with the addition of tricyclazole (Figures 18-20 left). Similar results were obtained from samples that were grown under tricyclazole conditions for seven days as were seen for the 14 day culture time. The presence of peaks of similar $\mathrm{m} / \mathrm{z}$ were observed from each spectra, however, the relative abundance of each peak was increased under tricyclazole conditions when compared to MEA only. The shorter growth duration, with the presence of tricyclazole, did however aid in the visualization of more peaks for A. niger. 


\section{Chapter IV}

\section{Discussion}

The necessity for rapid identification of microbial organisms for the successful treatment of patients with active infections has been shown in multiple studies. Current identification techniques, such as viable culture often require relatively long incubation periods, or have the potential for false-positive or false-negative results, as is the case for many current molecular and immunodiagnostic assays. Additionally, molecular and immunodiagnostic techniques have the capability to be rapid, however, there is a general lack of species-specific primers or monoclonal antibodies (19-24, 31). These techniques are used predominantly to detect the presence of a microorganism in a sample, not necessarily the identity, which is often required to determine the proper course of treatment. Relatively recently, MALDI-TOF MS, a rapid, non-biased, discriminate analytical technique has been shown to be a promising technique for fingerprinting microbial species. Specifically, MALDI-TOF MS has been used to identify clinically relevant bacteria and viral components, as well as fungi (43-62). Fungi are ubiquitous microorganisms capable of causing fatal invasive infections in immunocompromised individuals $(1,2)$. The successful treatment of invasive fungal infections is directly dependent on the rapid identification of the causal fungus and subsequent treatment of the patient with appropriate prophylactics (1).

Though MALDI-TOF MS has shown great promise in the utility of identifying fungi, one common problem has been reported by several groups when attempting to fingerprint darkly pigmented fungi. Darkly pigmented fungi commonly fail to produce a spectrum containing peaks that allow for their discrimination from other fungi $(64,73,75)$. Because darkly pigmented fungi have been isolated from contaminated indoor environments, and have been implicated in a vast array of diseases, these fungi are medically important, and their rapid identification is necessary for the successful treatment of disease. Therefore, the purpose of this study was to identify the cause for the inability to fingerprint darkly pigmented fungi, and to examine different methods that would allow for the fingerprinting of these organisms. 
One of the many purposes of fungal pigmentation, such as is present in darkly pigmented fungi, is to protect the fungus from UV light (77-79); therefore, it was hypothesized that dark fungal pigments were preventing the analytes in the MALDI-TOF MS preparation from forming peaks in the spectrum potentially by absorbing energy from the UV laser, used to induce ionization of the analyte, during MALDI-TOF MS analysis. In order to address this hypothesis, HSA was spiked with synthetic melanin and then analyzed for the $66 \mathrm{~m} / \mathrm{z}$ HSA peak. It was determined that the presence of high concentrations of synthetic melanin inhibited the formation of the spectra, as the control HSA only $66 \mathrm{~m} / \mathrm{z}$ peak height was reduced by $100 \%$ for concentrated synthetic melanin samples.

Because the synthetic melanin examined in the preceding experiments is not chemically identical to those obtained from fungal sources, fungal pigments from three different species of Aspergillus were isolated to determine whether these naturally obtained fungal pigments would also suppress $\mathrm{HSA}[\mathrm{M}+\mathrm{H}]^{+}$ ion formation in MALDI-TOF mass spectra. Fungal pigments were extracted from A. niger, a darkly pigmented fungus which was unable to be fingerprinted previously by multiple laboratories, A. fumigatus, which contains high concentrations of DHN- melanins yet still formed a unique spectrum. A. terreus, a fungus which is light brown when grown under sporulating conditions and also produced a unique spectrum. Before MALDI-TOF analysis was conducted with these samples, the pigment extracts were characterized. Pigments extracts from A. terreus appeared most like that of the synthetic melanin, whereas A. niger and A. fumigatus pigments contained cell wall components and maintained the shape of the spores from which they were extracted. In addition, each sample contained similar quantities of carbon, hydrogen, nitrogen, and oxygen, while none contained sulfur. This information helped to identify these pigments as potential eumelanins, as opposed to phaeomelanins, which contain sulfur $(80,84)$.

Furthermore, $1 \mathrm{mg} / \mathrm{mL}$ of each pigment exhibited strong absorption in the $200-800 \mathrm{~nm}$ range when analyzed by UV/VIS spectroscopy. Absorption for each pigment approached 1.0 at 337- nm which is the emission wavelength of the nitrogen laser used for MALDI-TOF MS. This is consistent with previous studies that have demonstrated the photoprotective properties of melanin in fungi, whereby melanins absorb UV radiation and dissipate this energy as heat via rapid internal conversion (84-86). 
Although the precise mechanism(s) by which the MALDI desorption/ionization event proceeds remains the subject of much discussion and active research (39), it is clear that the unique properties of melanin could interfere in a number of fundamental ways with the MALDI process. One plausible explanation for the observed inhibition of MALDI-TOF ionization is that melanin in fungal samples (particularly at high concentrations) directly competes with the organic acid matrix for irradiating photons. Following the MALDI laser pulse, ionization in the dense plume above the sample deposit will only occur where there is a sufficient population of electronically excited matrix molecules. If a photoexcitation/pooling mechanism for MALDI is assumed (76), mobile excitons in the bulk sample deposit could be quenched by melanin.

To demonstrate that melanin influences MALDI-TOF MS ionization, HSA was spiked with different concentrations of each of these fungal pigment extracts and the $66 \mathrm{~m} / \mathrm{z}$ peak height was analyzed. The presence of pigments from each fungus directly inhibited the $66 \mathrm{~m} / \mathrm{z}$ peak height formation, as greater than $89 \%$ of the $66 \mathrm{~m} / \mathrm{z}$ peak height was inhibited in the most concentrated samples of each pigment. Even at $0.001 \mathrm{mg} / \mathrm{mL}$ concentration of each pigment, with the exception of A. niger, the HSA peak was inhibited by greater than $10 \%$. Surprisingly, the pigment isolated from A. terreus, which produces a unique fingerprint spectrum, inhibited HSA peak formation by approximately $23 \%$ at the lowest concentration. To understand the influence of melanin on the sample spot, sample spots from each pigment preparation were visualized by microscopy. The results of these studies demonstrated that the pigment in each sample spot was not homogenously distributed. As shown by the FESEM data, the $A$. terreus pigment extract appeared as crystals of pigment and did not maintain the ultrastructural morphology of the spore wall as observed with A. fumigatus and A. niger. Therefore, it is possible that the pigment extracted from A. terreus was more homogenously distributed throughout the sample spot, and that the probability of the 90 laser strikes, which are culminated during each MALDI-TOF MS analysis, were present in more of the independent spectra obtained during each laser strike than in those obtained from A. fumigatus and A. niger. In order to address the hypothesis that the heterogeneity of the spot would alter the mass spectrum, MS equipped with a MALDI source was used to analyze angiotensin II which was spiked with A. fumigatus pigment preparation. Using this method, a single spectrum was obtained 
from an area within the sample spot, as directed by the user. It was determined that the angiotensin II peak height was greatly reduced in an area containing visible pigment as opposed to an area within the same spot that did not contain pigment. This evidence directly shows the ability of pigment to prevent the formation of peaks within a sample.

In order to determine if darkly pigmented fungi could be fingerprinted if pigmentation was not present, several culture techniques were used to produce fungi which lack pigmentation. The first method used was by growing the cultures under submerged conditions, during which the fungi do not produce pigment. These samples were analyzed and found to produce unique fingerprints which allowed them to be discriminated in canonical plots. Discrimination was based on the presence of 5 unique peaks that were discernable based on their relative intensities. These growth conditions were not the same as those which had been used to acquire previous fingerprinting data, as these conditions induced the formation of hyphae, as opposed to sporulation.

To further explore the influence of demelanizing fungal spores, fungal cultures were grown on MEA agar in the presence of tricyclazole, an antifungal agent known to specifically inhibit the formation of DHN-melanin. DHN-melanin has been reported to be present in A. fumigatus and potentially A. niger. When grown in the presence of tricyclazole, A. fumigatus changes from a dark green color to a light brown color, closer to that of A. terreus; however, A. niger did not change color. Despite the difference in color changes between the two fungi, the presence of tricyclazole increased the peak intensities for both A. fumigatus and A. terreus, but more importantly, resulted in the formation of 2 distinct peaks were observed for A. niger. Furthermore, pigmentation increases with the culture age, therefore fungi grown for 7 days instead of 14 days formed spectra that contained a greater paucity of peaks with higher relative abundance than those grown for 14 days under all conditions. In addition, A. niger produced a unique fingerprint spectrum at 7 days when grown on MEA only which was not observed in 14 day cultures.

In conclusion, fungal melanin suppresses peptide and protein ion signals during the acquisition of MALDI-TOF mass spectra. Suppression of peptide and protein standard ionization was observed upon addition of both commercially available synthetic melanin and melanins purified from three species of 
Aspergillus to the MALDI sample. Suppression of peptide and protein $[\mathrm{M}+\mathrm{H}]^{+}$ion signals was observed at melanin concentrations greater than $500 \mathrm{ng} / \mu \mathrm{L}$, a concentration significantly less than the level of melanin obtained from a fungal culture. Ion suppression occurs in a concentration dependent manner, approaching $100 \%$ at high melanin concentrations $(10 \mu \mathrm{g} / \mu \mathrm{L})$. Culture conditions may be manipulated to block fungal melanin synthesis and obtain acceptable MALDI-TOF fingerprint mass spectra from darkly pigmented species such as A. niger. MALDI-TOF fingerprint mass spectra were acquired from Aspergilli grown under liquid culture conditions, as well as cultures grown on malt extract agar supplemented with $50 \mu \mathrm{L} / \mathrm{mL}$ of tricyclazole, an antifungal agent used to block DHN-melanin synthesis.

To our knowledge, this is the first report demonstrating the detrimental effects of fungal pigments on the acquired MALDI-TOF mass spectrum, as well as the first report of successful fingerprinting of darkly pigmented fungi such as A. niger. This data represents an important step forward for the use of MALDI-TOF MS for the rapid identification of darkly pigmented fungi. In addition, these techniques may be used to aid in the identification of specific biomarkers for medically important fungi. With the identification of species-specific biomarkers via MALDI-TOF MS and subsequent sequencing and identification via MS/MS, recombinant proteins based on these biomarkers may be made and used to immunize mice for the production of monoclonal antibodies. Those antibodies may be exceptionally beneficial in the development of rapid, species-specific immunodiagnostic assays. 


\section{REFERENCES}

1. Latgé, J. P. 1999. Aspergillus fumigatus and aspergillosis. Clinical Microbiology Reviews 12:310350.

2. Eduard, W. 2009. Fungal spores: a critical review of the toxicological and epidemiological evidence as a basis for occupational exposure limit setting. Critical Reviews in Toxicology 39:799-864.

3. Ghorai, S., S. P. Banik, D. Verma, S. Chowdhury, S. Mukherjee, and S. Khowala. Fungal biotechnology in food and feed processing. Food Research International 42:577-587.

4. Broadbent, D. 1966. Antibiotics Produced by Fungi. Botanical Review 32:219-242.

5. Barrios-Gonzlez, J., and R. Miranda. Biotechnological production and applications of statins. Applied microbiology and biotechnology 85:869-883.

6. Crameri, R., M. Weichel, S. Flückiger, A. G. Glaser, and C. Rhyner. 2006. Fungal allergies: a yet unsolved problem. Chemical Immunology and Allergy 91:121-133.

7. Hogaboam, C. M., K. J. Carpenter, J. M. Schuh, and K. F. Buckland. 2005. Aspergillus and asthma--any link? Medical Mycology 43 Suppl 1:S197-202.

8. Kurup, V. P., H. D. Shen, and B. Banerjee. 2000. Respiratory fungal allergy. Microbes and Infection 2:1101-1110.

9. Portnoy, J. M., C. S. Barnes, and K. Kennedy. 2008. Importance of mold allergy in asthma. Current Allergy and Asthma Reports 8:71-78.

10. Eduard, W. 2006. The Nordic Expert Group for Criteria Documentation of Health Risks from Chemicals 139. Fungal Spores. In Arbete Och Halsa. 1-145.

11. Curtis, L., A. Lieberman, M. Stark, W. Rea, and M. Vetter. 2004. Adverse Health Effects of Indoor Molds. Journal of Nutritional \& Environmental Medicine 14:261-274.

12. Shelton, B., K. Kirkland, W. D. Flanders, and G. Morris. 2002. Profiles of airborne fungi in buildings and outdoor environments in the United States. Applied and Environmental Microbiology 68:1743-1753.

13. Kamei, K., and A. Watanabe. 2005. Aspergillus mycotoxins and their effect on the host. Medical Mycology 43 Suppl 1:S95-99.

14. Kurup, V. P., and G. Grunig. 2002. Animal models of allergic bronchopulmonary aspergillosis. Mycopathologia 153:165-177.

15. Romani, L. 2004. Immunity to fungal infections. Nature Reviews. Immunology 4:1-23.

16. Latge, J. P. 2001. The pathobiology of Aspergillus fumigatus. Trends in Microbiology 9:382-389.

17. Gullo, A. 2009. Invasive fungal infections: the challenge continues. Drugs 69 Suppl 1:65-73.

18. von Eiff, M., N. Roos, R. Schulten, M. Hesse, M. Zhlsdorf, and J. van de Loo. 1995. Pulmonary aspergillosis: early diagnosis improves survival. Respiration 62:341-347.

19. Wengenack, N., and M. Binnicker. 2009. Fungal molecular diagnostics. Clinics in Chest Medicine 30:391-408, viii.

20. Weile, J., and C. Knabbe. 2009. Current applications and future trends of molecular diagnostics in clinical bacteriology. Analytical and Bioanalytical Chemistry 394:731-742.

21. Boonsarngsuk, V., A. Niyompattama, C. Teosirimongkol, and K. Sriwanichrak. 2010. Falsepositive serum and bronchoalveolar lavage Aspergillus galactomannan assays caused by different antibiotics. Scandinavian Journal of Infectious Diseases 42:461-468.

22. Hope, W. W., T. J. Walsh, and D. W. Denning. 2005. Laboratory diagnosis of invasive aspergillosis. The Lancet Infectious Diseases 5:609-622.

23. Jones, M. E., A. J. Fox, A. J. Barnes, B. A. Oppenheim, P. Balagopal, G. R. Morgenstern, and J. H. Scarffe. 1998. PCR-ELISA for the early diagnosis of invasive pulmonary Aspergillus infection in neutropenic patients. Journal of Clinical Pathology 51:652-656.

24. Scotter, J., P. Campbell, T. Anderson, D. Murdoch, S. Chambers, and W. N. Patton. 2005. Comparison of PCR-ELISA and galactomannan detection for the diagnosis of invasive aspergillosis. Pathology 37:246-253. 
25. Scotter, J., and S. Chambers. 2005. Comparison of galactomannan detection, PCR-enzyme-linked immunosorbent assay, and real-time PCR for diagnosis of invasive aspergillosis in a neutropenic rat model and effect of caspofungin acetate. Clinical and Diagnostic Laboratory Immunology 12:1322-1327.

26. Thornton, C. 2010. Detection of invasive aspergillosis. Advances in Applied Microbiology 70:187-216.

27. Arruda, L. K., T. A. Platts-Mills, J. W. Fox, and M. D. Chapman. 1990. Aspergillus fumigatus allergen I, a major IgE-binding protein, is a member of the mitogillin family of cytotoxins. The Journal of Experimental Medicine 172:1529-1532.

28. Weig, M., M. Frosch, K. Tintelnot, A. Haas, U. Gross, B. Linsmeier, and J. Heesemann. 2001. Use of recombinant mitogillin for improved serodiagnosis of Aspergillus fumigatus-associated diseases. Journal of clinical microbiology 39:1721-1730.

29. Hohl, T. M., and M. Feldmesser. 2007. Aspergillus fumigatus: principles of pathogenesis and host defense. Eukaryotic Cell 6:1953-1963.

30. Preuner, S., and T. Lion. 2009. Towards molecular diagnostics of invasive fungal infections. Expert Review of Molecular Diagnostics 9:397-401.

31. Wheat, L. J. 2009. Approach to the diagnosis of the endemic mycoses. Clinics in Chest Medicine 30:379-389, viii.

32. Khot, P., and D. Fredricks. 2009. PCR-based diagnosis of human fungal infections. Expert Review of Anti-Infective Therapy 7:1201-1221.

33. Leski, T., A. Malanoski, D. Stenger, and B. Lin. 2010. Target amplification for broad spectrum microbial diagnostics and detection. Future Microbiology 5:191-203.

34. Hawksworth, D. L. 1991. The fungal dimension of biodiversity: magnitude, significance, and conservation. Mycological Research 95:641-655.

35. Guarro, J., J. Gene, and A. M. Stchigel. 1999. Developments in Fungal Taxonomy. Clinical Microbiology Reviews 12:454-500.

36. Hillenkamp, F., and M. Karas. 1990. Mass spectrometry of peptides and proteins by matrixassisted ultraviolet laser desorption/ionization. Methods Enzymol 193:280-295.

37. Karas, M., and F. Hillenkamp. 1988. Laser desorption ionization of proteins with molecular masses exceeding 10,000 daltons. Analytical Chemistry 60:2299-2301.

38. Frankevich, V., J. Zhang, S. Friess, M. Dashtiev, and R. Zenobi. 2003. Role of electrons in laser desorption/ionization mass spectrometry. Analytical Chemistry 75:6063-6067.

39. Knochenmuss, R. 2006. Ion formation mechanisms in UV-MALDI. The Analyst 131:966-986.

40. Knochenmuss, R., and R. Zenobi. 2003. MALDI ionization: the role of in-plume processes. Chemical Reviews 103:441-452.

41. Cotter, R. J. 1999. The new time-of-flight mass spectrometry. Analytical Chemistry 71:445A451A.

42. Cotter Robert, J. 2009. Time-of-Flight Mass Spectrometry. In Time-of-Flight Mass Spectrometry. American Chemical Society. 16-48.

43. Teresa, C. C., M. L. David, J. W. Walter, Jr., and A. Vertes. 1994. Differentiation of bacteria using protein profiles from matrix-assisted laser desorption/ionization time-of-flight mass spectrometry. Rapid Communications in Mass Spectrometry 8:1026-1030.

44. Claydon, M. A., S. N. Davey, V. Edwards-Jones, and D. B. Gordon. 1996. The rapid identification of intact microorganisms using mass spectrometry. Nature Biotechnology 14:15841586.

45. Holland, R. D., J. G. Wilkes, F. Rafii, J. B. Sutherland, C. C. Persons, K. J. Voorhees, and J. O. Lay. 1996. Rapid identification of intact whole bacteria based on spectral patterns using matrixassisted laser desorption/ionization with time-of-flight mass spectrometry. Rapid Communications in Mass Spectrometry 10:1227-1232. 
46. Krishnamurthy, T., and P. L. Ross. 1996. Rapid identification of bacteria by direct matrix-assisted laser desorption/ionization mass spectrometric analysis of whole cells. Rapid Communications in Mass Spectrometry 10:1992-1996.

47. Giebel, R., C. Worden, S. M. Rust, G. T. Kleinheinz, M. Robbins, and T. R. Sandrin. 2010. Microbial fingerprinting using matrix-assisted laser desorption ionization time-of-flight mass spectrometry (MALDI-TOF MS) applications and challenges. Advances in Applied Microbiology 71:149-184.

48. Bernardo, K., N. Pakulat, M. Macht, O. Krut, H. Seifert, S. Fleer, F. Hnger, and M. Krnke. 2002. Identification and discrimination of Staphylococcus aureus strains using matrix-assisted laser desorption/ionization-time of flight mass spectrometry. Proteomics 2:747-753.

49. Hettick, J., M. Kashon, J. Slaven, Y. Ma, J. Simpson, P. Siegel, G. Mazurek, and D. Weissman. 2006. Discrimination of intact mycobacteria at the strain level: a combined MALDI-TOF MS and biostatistical analysis. Proteomics 6:6416-6425.

50. Bizzini, A., C. Durussel, J. Bille, G. Greub, and G. Prod'hom. Performance of Matrix-Assisted Laser Desorption Ionization-Time of Flight Mass Spectrometry for Identification of Bacterial Strains Routinely Isolated in a Clinical Microbiology Laboratory. Journal of Clinical Microbiology 48:1549-1554.

51. Christner, M., H. Rohde, M. Wolters, I. Sobottka, K. Wegscheider, and M. Aepfelbacher. Rapid identification of bacteria from positive blood culture bottles by use of matrix-assisted laser desorption-ionization time of flight mass spectrometry fingerprinting. Journal of Clinical Microbiology 48:1584-1591.

52. $\quad$ Ferreira, L., S. Vega, F. Sánchez-Juanes, M. González, A. Herrero, M. C. Muñiz, J. M. GonzálezBuitrago, and J. L. Muñoz. Identificación bacteriana mediante espectrometría de masas matrixassisted laser desorption ionization time-of-flight. Comparación con la metodología habitual en los laboratorios de Microbiología Clínica. Enfermedades Infecciosas y Microbiología Clínica In Press, Corrected Proof.

53. Seng, P., M. Drancourt, F. Gouriet, B. La Scola, P.-E. Fournier, Jean M. Rolain, and D. Raoult. 2009. Ongoing Revolution in Bacteriology: Routine Identification of Bacteria by Matrix-Assisted Laser Desorption Ionization Time-of-Flight Mass Spectrometry. Clinical Infectious Diseases 49:543-551.

54. van Veen, S. Q., E. C. J. Claas, and E. Kuijper. High-throughput identification of bacteria and yeast by matrix-assisted laser desorption ionization-time of flight mass spectrometry in conventional medical microbiology laboratories. Journal of Clinical Microbiology 48:900-907.

55. Hettick, J., M. Kashon, J. Simpson, P. Siegel, G. Mazurek, and D. Weissman. 2004. Proteomic profiling of intact mycobacteria by matrix-assisted laser desorption/ionization time-of-flight mass spectrometry. Analytical Chemistry 76:5769-5776.

56. Colquhoun, D., K. Schwab, R. Cole, and R. Halden. 2006. Detection of norovirus capsid protein in authentic standards and in stool extracts by matrix-assisted laser desorption ionization and nanospray mass spectrometry. Applied and Environmental Microbiology 72:2749-2755.

57. Lopaticki, S., C. J. Morrow, and J. J. Gorman. 1998. Characterization of pathotype-specific epitopes of newcastle disease virus fusion glycoproteins by matrix-assisted laser desorption/ionization time-of-flight mass spectrometry and post-source decay sequencing. Journal of Mass Spectrometry 33:950-960.

58. Parker, C. E., D. I. Papac, and K. B. Tomer. 1996. Monitoring Cleavage of Fusion Proteins by Matrix-Assisted Laser Desorption Ionization/Mass Spectrometry: Recombinant HIV-1IIIBp26. Analytical Biochemistry 239:25-34.

59. Kim, Y. J., A. Freas, and C. Fenselau. 2001. Analysis of Viral Glycoproteins by MALDI-TOF Mass Spectrometry. Analytical Chemistry 73:1544-1548.

60. Ilina, E., M. Malakhova, E. Generozov, E. Nikolaev, and V. Govorun. 2005. Matrix-assisted laser desorption ionization-time of flight (mass spectrometry) for hepatitis $\mathrm{C}$ virus genotyping. Journal of Clinical Microbiology 43:2810-2815. 
61. Luan, J., J. Yuan, X. Li, S. Jin, L. Yu, M. Liao, H. Zhang, C. Xu, Q. He, B. Wen, X. Zhong, X. Chen, H. L. Y. Chan, J. J. Y. Sung, B. Zhou, and C. Ding. 2009. Multiplex detection of 60 hepatitis B virus variants by MALDI-TOF mass spectrometry. Clinical Chemistry 55:1503-1509.

62. Michael, K., T. Harder, T. Mettenleiter, and A. Karger. 2009. Diagnosis and strain differentiation of avian influenza viruses by restriction fragment mass analysis. Journal of Virological Methods 158:63-69.

63. Valentine, N., S. Wunschel, D. Wunschel, C. Petersen, and K. Wahl. 2005. Effect of culture conditions on microorganism identification by matrix-assisted laser desorption ionization mass spectrometry. Applied and Environmental Microbiology 71:58-64.

64. Hettick, J., B. Green, A. Buskirk, M. Kashon, J. Slaven, E. Janotka, F. Blachere, D. Schmechel, and D. Beezhold. 2008. Discrimination of Aspergillus isolates at the species and strain level by matrix-assisted laser desorption/ionization time-of-flight mass spectrometry fingerprinting. Analytical Biochemistry 380:276-281.

65. Hettick, J., B. Green, A. Buskirk, M. Kashon, J. Slaven, E. Janotka, F. Blachere, D. Schmechel, and D. Beezhold. 2008. Discrimination of Penicillium isolates by matrix-assisted laser desorption/ionization time-of-flight mass spectrometry fingerprinting. Rapid Communications in Mass Spectrometry 22:2555-2560.

66. Hsieh, S.-Y., C.-L. Tseng, Y.-S. Lee, A.-J. Kuo, C.-F. Sun, Y.-H. Lin, and J.-K. Chen. 2008. Highly efficient classification and identification of human pathogenic bacteria by MALDI-TOF MS. Molecular \& Cellular Proteomics 7:448-456.

67. Arnold, R. J., J. A. Karty, A. D. Ellington, and J. P. Reilly. 1999. Monitoring the growth of a bacteria culture by MALDI-MS of whole cells. Analytical Chemistry 71:1990-1996.

68. Saenz, A. J., C. E. Petersen, N. B. Valentine, S. L. Gantt, K. H. Jarman, M. T. Kingsley, and K. L. Wahl. 1999. Reproducibility of matrix-assisted laser desorption/ionization time-of-flight mass spectrometry for replicate bacterial culture analysis. Rapid Communications in Mass Spectrometry 13:1580-1585.

69. Smole, S., L. King, P. Leopold, and R. Arbeit. 2002. Sample preparation of Gram-positive bacteria for identification by matrix assisted laser desorption/ionization time-of-flight. Journal of Microbiological Methods 48:107-115.

70. Walker, J., A. J. Fox, V. Edwards-Jones, and D. B. Gordon. 2002. Intact cell mass spectrometry (ICMS) used to type methicillin-resistant Staphylococcus aureus: media effects and interlaboratory reproducibility. Journal of Microbiological Methods 48:117-126.

71. Wunschel, S. C., K. H. Jarman, C. E. Petersen, N. B. Valentine, K. L. Wahl, D. Schauki, J. Jackman, C. P. Nelson, and E. White V. 2005. Bacterial analysis by MALDI-TOF mass spectrometry: An inter-laboratory comparison. Journal of the American Society for Mass Spectrometry 16:456-462.

72. Bessède, E., M. Angla-gre, Y. Delagarde, S. S. Hieng, A. Ménard, and F. Mégraud. Matrixassisted laser-desorption/ionization biotyper: experience in the routine of a University hospital. Clinical Microbiology and Infection 9999.

73. Li, T. Y., B. H. Liu, and Y. C. Chen. 2000. Characterization of Aspergillus spores by matrixassisted laser desorption/ionization time-of-flight mass spectrometry. Rapid Communications in Mass Spectrometry 14:2393-2400.

74. Amiri-Eliasi, B., and C. Fenselau. 2001. Characterization of protein biomarkers desorbed by MALDI from whole fungal cells. Analytical Chemistry 73:5228-5231.

75. Valentine, N., J. Wahl, M. Kingsley, and K. Wahl. 2002. Direct surface analysis of fungal species by matrix-assisted laser desorption/ionization mass spectrometry. Rapid Communications in Mass Spectrometry 16:1352-1357.

76. Ehring, H., M. Karas, and F. Hillenkamp. 1992. Role of photoionization and photochemistry in ionization processes of organic molecules and relevance for matrix-assisted laser desorption lonization mass spectrometry. Organic Mass Spectrometry 27:472-480. 
77. Brakhage, A. A., K. Langfelder, G. Wanner, A. Schmidt, and B. Jahn. 1999. Pigment biosynthesis and virulence. Contributions to Microbiology 2:205-215.

78. Wheeler, M. H., and A. A. Bell. 1988. Melanins and their importance in pathogenic fungi. Current Topics in Medical Mycology 2:338-387.

79. Jacobson, E. S. 2000. Pathogenic roles for fungal melanins. Clinical Microbiology Reviews 13:708-717.

80. Hamilton, A. J., and B. L. Gomez. 2002. Melanins in fungal pathogens. Journal of Medical Microbiology 51:189-191.

81. Henson, J., M. Butler, and A. Day. 1999. THE DARK SIDE OF THE MYCELIUM: Melanins of Phytopathogenic Fungi. Annual Review of Phytopathology 37:447-471.

82. Langfelder, K., M. Streibel, B. Jahn, G. Haase, and A. Brakhage. 2003. Biosynthesis of fungal melanins and their importance for human pathogenic fungi. Fungal Genetics and Biology 38:143158.

83. Gomez, B., and J. Nosanchuk. 2003. Melanin and fungi. Current opinion in infectious diseases 16:91-96.

84. Gomez, B., and J. Nosanchuk. 2003. Melanin and fungi. Current Opinion in Infectious Diseases 16:91-96.

85. Plonka, P., and M. Grabacka. 2006. Melanin synthesis in microorganisms--biotechnological and medical aspects. Acta Biochimica Polonica 53:429-443.

86. Butler, M. J., A. W. Day, J. M. Henson, and N. P. Money. 2001. Pathogenic Properties of Fungal Melanins. Mycologia 93:1-8.

87. Seraglia, R., P. Traldi, G. Elli, A. Bertazzo, C. Costa, and G. Allegri. 1993. Laser desorption ionization mass spectrometry in the study of natural and synthetic melanins. I--Tyrosine melanins. Biological Mass Spectrometry 22:687-697.

88. Fogarty, R. V., and J. M. Tobin. 1996. Fungal melanins and their interactions with metals. Enzyme and Microbial Technology 19:311-317.

89. Nosanchuk, J., and A. Casadevall. 2003. Budding of melanized Cryptococcus neoformans in the presence or absence of L-dopa. Microbiology 149:1945-1951.

90. Lee, J.-K., H.-M. Jung, and S.-Y. Kim. 2003. 1,8-dihydroxynaphthalene (DHN)-melanin biosynthesis inhibitors increase erythritol production in Torula corallina, and DHN-melanin inhibits erythrose reductase. Applied and Environmental Microbiology 69:3427-3434.

91. Wang, Y., P. Aisen, and A. Casadevall. 1996. Melanin, melanin "ghosts," and melanin composition in Cryptococcus neoformans. Infection and Immunity 64:2420-2424.

92. Hettick, J. M., B.J. Green, A.D. Buskirk, J.E. Slaven, M.L. Kashon, and D. H. Beezhold. 2010. Discrimination of fungi by MALDI-TOF mass spectrometry. In Rapid Characterization of Microorganisms by Mass Spectrometry. E. P. Demirev and C. Fenselau, ed.

93. Eisenman, H., J. Nosanchuk, J. B. W. Webber, R. Emerson, T. Camesano, and A. Casadevall. 2005. Microstructure of cell wall-associated melanin in the human pathogenic fungus Cryptococcus neoformans. Biochemistry 44:3683-3693.

94. Hettick, J. M., D. L. McCurdy, D. C. Barbacci, and D. H. Russell. 2001. Optimization of sample preparation for peptide sequencing by MALDI-TOF photofragment mass spectrometry. Analytical Chemistry 73:5378-5386.

95. Koomen, J. M., W. K. Russell, J. M. Hettick, and D. H. Russell. 2000. Improvement of resolution, mass accuracy, and reproducibility in reflected mode DE-MALDI-TOF analysis of DNA using fast evaporation--overlayer sample preparations. Analytical Chemistry 72:3860-3866.

96. Tsai, H. F., M. H. Wheeler, Y. C. Chang, and K. J. Kwon-Chung. 1999. A developmentally regulated gene cluster involved in conidial pigment biosynthesis in Aspergillus fumigatus. Journal of Bacteriology 181:6469-6477. 


\section{CURRICULUM VITAE}

\section{Amanda D. Buskirk}

16117 Old Beechwood RD SW • Lonaconing, MD $21539 \diamond$ Phone (301) 697-0537

• Email: abuskirk@mix.wvu.edu

\section{EDUCATION}

M.S. in Biomedical Sciences

In progress

West Virginia University, Morgantown, WV

GPA 3.2/4.0

Advisor: Dr. Donald Beezhold

B.S. in Biology, concentration in Biotechnology

December 2004

Minor in Chemistry

GPA 3.4/4.0

Frostburg State University Frostburg, MD

\section{AWARDS}

West Virginia University Graduate Travel Award

HOPE Science and Technology Scholarship

2001-2004

Academic Excellence Scholarship

2001-2004

Lewis J. Ort Scholarship

2003

Hadley and Smouse Scholarship

2003

\section{POSITIONS HELD}

Graduate Researcher, West Virginia University, Morgantown, WV

Member of the Allergy and Clinical Immunology Branch

at CDC/NIOSH utilizing mass spectrometry to fingerprint mycobacteria

and fungal isolates, as well as developing a murine model of dry

mycoaerosol exposures and characterizing the resulting

immune response using histology, flow cytometry, and immunoassays.

Research Assistant I, Windber Research Institute, Windber, PA

March-June 2005

Member of the proteomics laboratory working with preparation of

breast cancer samples for 2-DIGE and subsequent mass

spectrometry analysis searching for unique biomarkers.

Student Intern, Windber Research Institute, Windber, PA

Summer 2004

Member of the proteomics laboratory performing western blot

analysis on breast cancer samples.

\section{PRESENTATIONS}

$110^{\text {th }}$ General Meeting of the American Society for Microbiology, San Diego, CA

June 2005-present

"Murine Pulmonary CD4 and CD8 T-cell Responses to Aspergillus conidia are Species-Dependent"

Van Liere Convocation and Research Day, Morgantown, WV

April 2009

* "Developing and Characterizing a Mouse Model of Dry Mycoaerosol Exposures"

Joint Statistical Meeting, Denver, CO

"Classification of Penicillium Strains Using Discriminant Analysis" 
$\mathbf{5 6}^{\text {th }}$ ASMS Conference on Mass Spectrometry and Allied Topics, Denver, CO

June 2008

"Discrimination of Penicillium isolates by Matrix Assisted Laser Desorption/Ionization Time- of- Flight

Mass Spectrometry Fingerprinting"

56 $^{\text {th }}$ ASMS Conference on Mass Spectrometry and Allied Topics, Denver, CO

June 2008

* "Discrimination of Aspergillus isolates at the species and strain level by MALDI-TOF mass spectrometry fingerprinting"

Van Liere Convocation and Research Day, Morgantown, WV

April 2008

* "Developing and Characterizing a Mouse Model Of Fungal Exposure To Dry Spores"

Van Liere Convocation and Research Day, Morgantown, WV

*"Optimization of MALDI-TOF Mass Spectrometry Analysis of Mycobacteria"

April 2007

American Academy of Asthma Allergy and Immunology, Philadelphia, PA

March 2008

"Identifying fungal isolates using matrix-assisted laser desorption/ionization time-of-flight mass

spectrometry"

$55^{\text {th }}$ ASMS Conference on Mass Spectrometry and Allied Topics, Indianapolis, ID

June 2007

* "Investigation of Sample Preparation Strategies for MALDI- TOF MS Analysis

of Mycobacterial Proteins"

$55^{\text {th }}$ ASMS Conference on Mass Spectrometry and Allied Topics, Indianapolis, ID

June 2007

"MALDI-TOF MS Fingerprinting of Fungal Isolates"

$\mathbf{5 5}^{\text {th }}$ ASMS Conference on Mass Spectrometry and Allied Topics, Indianapolis, ID

June 2007

"Identification of Mycobacteria and Mycobacteria Biomarker Proteins by Novel

Biological Preparation Combined with Tandem Mass Spectrometry"

NOTE: * Denotes first author posters

\section{PUBLICATIONS}

A.D. Buskirk, B.J. Green, J.M. Hettick, I. Chipinda, B.F. Law, P.D. Siegel, J.E. Slaven, and D.H. Beezhold. "Fungal pigments inhibit the MALDI-TOF mass spectrometry analysis of darkly pigmented fungi". Analytical Biochemistry. 2010. Submitted.

S.P. Templeton, A.D. Buskirk, B. Law, B.J. Green, and D.H. Beezhold. "Aspergillus Conidia Germination Enhances Murine Airway $\mathrm{CD}^{+}$T-cell Responses". Manuscript in preparation.

J.M. Hettick, B.J. Green, A.D. Buskirk, J.E. Slaven, M.L. Kashon, and D.H. Beezhold. "Discrimination of fungi by MALDI-TOF mass spectrometry". Rapid Characterization of Microorganisms by Mass Spectrometry, P. Demirev and C. Fenselau, Eds. 2010. In press.

S.P. Templeton, A.D. Buskirk, B.J. Green, D.H. Beezhold, D. Schmechel. "Murine Models of Airway Fungal Exposure and Allergic Sensitization". Medical Mycology. 2009. 48(2) 217-228.

J.M. Hettick, B.J. Green, A.D. Buskirk, M.L. Kashon, J.E. Slaven, E. Janotka, F.M. Blachere, D. Schmechel, and D.H. Beezhold. "Discrimination of Penicillium isolates by matrix-assisted laser desorption/ionization time-of-flight mass spectrometry fingerprinting." Rapid Communications in Mass Spectrometry 2008. (16) 2555-2560.

J.M. Hettick, B.J. Green, A.D. Buskirk, M.L. Kashon, J.E. Slaven, E. Janotka, F.M. Blachere, D. Schmechel, and D.H. Beezhold. "Identification of Aspergillus isolates at the species and strain level by matrix-assisted laser desorption/ionization time-of-flight mass spectrometry fingerprinting." Analytical. Biochemistry 2008. (380) 276-281. 